JOURNAL OF RESEARCH of the National Bureau of Standards - B. Mathematical Sciences

Vol. 73B, No. 2, April-June 1969

\title{
An Evaluation of Linear Least Squares Computer Programs
}

\author{
Roy H. Wampler
}

Institute for Basic Standards, National Bureau of Standards, Washington, D.C. 20234

(January 8, 1969)

\begin{abstract}
Two linear least squares test problems, both fifth degree polynomials, have been run on more than twenty different computer programs in order to assess their numerical accuracy. Among the programs tested were representatives from various statistical packages as well as some from the SHARE library. Essentially five different algorithms were used in the various programs to obtain the coefficients of the least squares fits. The tests were run on several different computers, in double precision as well as single precision. By comparing the coefficients reported, it was found that those programs using orthogonal Householder transformations or Gram-Schmidt orthonormalization were much more accurate than those using elimination algorithms. Programs using orthogonal polynomials (suitable only for polynomial fits) also proved to be superior to those using elimination algorithms. One program, using congruential methods and integer arithmetic, obtained exact solutions. In a number of programs, the coefficients reported in one test problem were sometimes completely erroneous, containing not even one correct significant digit.
\end{abstract}

Key words: Computer programs; Gram-Schmidt orthogonalization; Householder transformations; least squares; linear equations; orthogonalization; orthogonal polynomials; regression; rounding error; stepwise regression.

\section{Introduction}

Since the time when the electronic computer began to supplant the desk calculator as the chief tool for solving linear least squares problems, numerous least squares computer programs have been written. These programs have utilized a variety of computational algorithms. Because least squares problems are by their very nature frequently ill-conditioned, the numerical accuracy achieved by a least squares program strongly depends upon the choice of the algorithm. Many programs have been written which use methods appropriate for desk calculators but inappropriate for computers. Anscombe $[1]^{1}$ has aptly remarked: "Textbooks of statistical method display a wonderful unanimity in recommending computational procedures that are suited to desk calculators but are perilous for computers. Only with some determination can the statistician break himself of bad habits and become adequately informed about round-off error."

The present study was undertaken to assess the numerical accuracy of representative least squares programs from a variety of sources. Two test problems, both fifth degree polynomials, have been run on more than twenty different programs. Included in the study were programs from the BMD Biomedical Computer Programs collection, the C-E-I-R Multi-Access Computing Services library, the IBM SHARE library, the IBM System/360 Scientific Subroutine Package, the Univac MATH-PACK and STAT-PACK collections, and the Project MAC 7094 disk files. A detailed listing of the sources of the programs is given in appendix A, together with a brief description of each program.

For a number of programs, the test problems were run in double precision as well as in single precision. This, of course, necessitated certain changes in the original programs.

The programs included in this study used essentially five different algorithms: orthogonal

\footnotetext{
${ }^{1}$ Figures in brackets indicate the literature references at the end of this paper.
} 
Householder transformations, Gram-Schmidt orthonormalization, orthogonal polynomials, Gaussian or Jordan elimination, and a congruential method with computations in integer arithmetic.

Previous studies appraising linear least squares programs and comparing the results of different algorithms have been made by Cameron [9], Freund [20], Bright and Dawkins [7], Zellner and Thornber [46], Longley [29], and Jordan [27]. The present study differs from the earlier ones mainly by including a larger selection of widely used and easily accessible programs.

The linear least squares problem may be briefly stated as follows: One has $n$ observations or measurements of a "dependent" variable $y$ which are statistically independent with common variance $\sigma^{2}$ whose expected values are given by a linear function of the corresponding values of $k$ "independent" variables, $x_{1}, x_{2}, \ldots ., x_{k}, k \leq n$. In matrix notation we say that the $n$ observations have expected values $E(Y)=X \beta$, where $Y$ is an $n \times 1$ vector, $X$ is an $n \times k$ matrix, and $\beta$ is a $k \times 1$ vector of unknown coefficients. Assuming that $X$ is of rank $k$, the least squares estimates of the coefficients are given by $\hat{\beta}=\left(X^{\prime} X\right)^{-1} X^{\prime} Y$. Other quantities of interest are $\hat{Y}=X \hat{\beta}$, the vector of pre-

dicted values; $\delta=Y-\hat{Y}$, the vector of residuals; and $s^{2}=\frac{1}{n-k}(Y-\hat{Y})^{\prime}(Y-\hat{Y})$, an estimate of the variance $\sigma^{2}$.

In running certain programs, modifications were occasionally made to input and output formats. Other changes were made in five of the programs using elimination algorithms because the original versions of these programs failed to give solutions to the fifth degree polynomial problems. In particular, features that may have been intended to prevent execution of computations subject to excessive rounding error were sometimes bypassed. Details of these changes, and some remarks on the effectiveness of the features which were bypassed, will be given in section 8 .

Four computers were used: the GE 235, the IBM 7094, and the Univac 1107 and 1108. The 1108 which was used is located at the National Bureau of Standards, and the 7094 which was chiefly used is located at Harry Diamond Laboratories, Washington, D. C. The programs run on the 235, the 1107 and the Project MAC 7094 utilized consoles at the National Bureau of Standards connected to computers at other locations.

\section{The Test Problems}

The two main test problems which were used throughout this investigation are identified as $Y 1$ and $Y 2$. Both were fifth degree polynomials, with the values of $x$ being the integers $0,1,2, \ldots$, 20. The "observations," $Y 1$ and $Y 2$, were calculated from the following equations:

$$
\begin{aligned}
& Y 1: y=1+x+x^{2}+x^{3}+x^{4}+x^{5}, x=0(1) 20 \\
& Y 2: y=1+0.1 x+0.01 x^{2}+0.001 x^{3}+0.0001 x^{4}+0.00001 x^{5}, x=0(1) 20 .
\end{aligned}
$$

Thus the values of $Y 1$ were integers having from one to seven digits, and those of $Y 2$ were fivedecimal numbers ranging from 1.00000 to 63.00000 .

If the least squares solutions were computed with no rounding error, one would obtain

$$
\hat{\beta}(Y 1)=\left[\begin{array}{l}
1 \\
1 \\
1 \\
1 \\
1 \\
1
\end{array}\right], \quad \hat{\beta}(Y 2)=\left[\begin{array}{l}
1 . \\
0.1 \\
.01 \\
.001 \\
.0001 \\
.00001
\end{array}\right] \text {, }
$$

and for both problems the residual standard deviation would be zero.

For some programs the input required was the 21 values of $x$ and $y$. Some programs required, in addition, the powers $x^{2}, x^{3}, x^{4}$, and $x^{5}$ to be entered as input. Other programs required as input the 6 by 6 matrix $X^{\prime} X$ and the 6 by 1 vector $X^{\prime} Y$. It should be noted that the elements of $X^{\prime} X$ are 
integers having from 2 to 14 digits, the elements of $X^{\prime} Y$ for $Y 1$ are 8- to 14-digit integers, and the elements of $X^{\prime} Y$ for $Y 2$ are 5-decimal numbers having up to 13 significant digits. The input is listed in table 9.

The two test problems, $Y 1$ and $Y 2$, were chosen because they are so highly ill-conditioned that some programs fail to obtain correct solutions while other programs succeed in obtaining reasonably accurate solutions. Polynomial problems were chosen because polynomial fitting is an important type of linear least squares problem which occurs frequently in practice.

The ill-conditioning of the two test problems can be described more explicitly. One measure of the condition of a matrix $A$ is the $P$-condition, defined as

$$
P(A)=\left|\frac{\lambda}{\mu}\right|
$$

where $\lambda$ is the numerically largest eigenvalue of $A$ and $\mu$ is the numerically smallest eigenvalue of $A$. (See Newman [34, p. 240]).

For $A=X^{\prime} X$, the $6 \times 6$ matrix associated with $Y 1$ and $Y 2$, the $P$-condition is $4.095 \times 10^{13}$. In this respect, it is similar to the Hilbert matrix of order 10 , whose $P$-condition is $1.603 \times 10^{13}$ (see Fettis and Caslin [17]). The $P$-condition of the Hilbert matrix of order 11 is $5.231 \times 10^{14}$. The relation between the Hilbert matrix and the matrix $X^{\prime} X$ which arises in a polynomial fit is discussed in Forsythe [18].

Most of the programs which were tested obtained more accurate solutions for $Y 2$ than for $Y 1$. If we let $A$ denote the $7 \times 7$ matrix

$$
A=\left[\begin{array}{cc}
X^{\prime} X & X^{\prime} Y \\
Y^{\prime} X & 0
\end{array}\right]
$$

we find that for $Y 2, P(A)=4.095 \times 10^{13}$, whereas for $Y 1, P(A)=6.829 \times 10^{13}$, indicating that the system involving $Y 1$ is more ill-conditioned than that involving $Y 2$.

The test problem used by Longley [29] was also highly ill-conditioned. For the $7 \times 7$ matrix $X^{\prime} X$ of his problem, the $P$-condition is $2.361 \times 10^{19}$.

\section{Summary of the Results}

Tables 1 to 6 present a brief summary of the main results. A count, $C_{j}$, of the number of correct significant digits in each computed coefficient was obtained as follows:

Let $\beta_{j}(j=1,2, \ldots, 6)$ denote the "true" value of the coefficient - that is, the value computed with no rounding error. Let $\hat{\beta}_{j}$ denote the value calculated by the computer. Then

$C_{j}=\left\{\begin{array}{l}-\log _{10}\left|\frac{\beta_{j}-\hat{\beta}_{j}}{\beta_{j}}\right| \text {, if }\left|\beta_{j}-\hat{\beta}_{j}\right| \neq 0 \text { and } \beta_{j} \neq 0 \\ -\log _{10}\left|\beta_{j}-\hat{\beta}_{j}\right|, \text { if }\left|\beta_{j}-\hat{\beta}_{j}\right| \neq 0 \text { and } \beta_{j}=0 \\ D, \text { the approximate number of decimal digits with which the machine computes, if } \beta_{j}-\hat{\beta}_{j}=0 .\end{array}\right.$

The above approach to counting the number of correct digits in a computed value has been used by Jordan [27] and others.

Tables 1 to 6 , in the columns headed "Average Number of Correct Digits" report

$$
C=\frac{1}{6} \sum_{j=1}^{6} C_{j}
$$

From the above definition, a negative count can occur. For example, if $\beta_{j}=1.0$, and $\hat{\beta}_{j}=136.0$, we get $C_{j}=-2.130$. This indicates that $\hat{\beta}_{j}$ is wrong by roughly two orders of magnitude. 
TABLE 1. Summary of programs run in single precision -8 digits

\begin{tabular}{|c|c|c|c|c|c|c|c|c|}
\hline \multirow{2}{*}{ Program } & \multirow{2}{*}{$\begin{array}{l}\text { Com- } \\
\text { puter }\end{array}$} & \multirow{2}{*}{\begin{tabular}{|l} 
Algo- \\
rithm
\end{tabular}} & \multicolumn{2}{|c|}{$\begin{array}{l}\text { Average number of } \\
\text { correct digits }\end{array}$} & \multicolumn{2}{|c|}{ Rank } & \multicolumn{2}{|c|}{ Date of run } \\
\hline & & & $Y 1$ & $Y 2$ & $Y 1$ & $Y 2$ & $Y 1$ & $Y 2$ \\
\hline ALSQ $\ldots$ & 1108 & HT & 4.098 & 5.368 & 3 & 5 & $10-18-67$ & $10-18-67$ \\
\hline BMD02R. & 1108 & E & -0.106 & 1.981 & 12 & 14 & $12-13-67$ & $11-17-67$ \\
\hline BMD03R. & 7094 & $\mathrm{E}$ & 0.742 & 1.721 & 8 & 16 & $12-30-66$ & $1-3-67$ \\
\hline BMD03R. & 1108 & $\mathrm{E}$ & -0.123 & 2.287 & 13 & 12 & $12-18-67$ & $12-18-67$ \\
\hline DAM...... & 7094 & $\mathrm{E}$ & 1.389 & 2.312 & 7 & 11 & $4-12-67$ & $4-12-67$ \\
\hline DAM $\ldots \ldots \ldots \ldots \ldots$ & 1108 & $\mathrm{E}$ & -0.264 & 2.622 & 16 & 9 & $3-5-68$ & $3-5-68$ \\
\hline LINFIT (Miller).. & 7094 & $?$ & -2.756 & -0.301 & 21 & 21 & $5-17-68$ & $8-15-68$ \\
\hline LSTSQ ..................... & 1108 & HT & 4.528 & 5.840 & 1 & 3 & $5-1-68$ & $5-6-68$ \\
\hline MATH-PACK, ORTHLS. & 1108 & $\mathrm{OP}$ & 2.118 & 4.363 & 6 & 6 & $4-12-68$ & $4-12-68$ \\
\hline MPR3 $\ldots \ldots \ldots \ldots \ldots \ldots \ldots$ & 7094 & $\mathrm{E}$ & -0.140 & 1.856 & 14 & 15 & $5-16-67$ & $5-18-67$ \\
\hline UMNITAB (Invert).. & 7094 & $\mathrm{E}$ & -0.607 & 1.460 & 17 & 18 & $12-9-66$ & $12-9-66$ \\
\hline OMNITAB (Invert)... & 1108 & $\mathrm{E}$ & -0.907 & 1.224 & 19 & 19 & $2-29-68$ & $2-29-68$ \\
\hline OMNITAB (Ortho)... & 7094 & GS & 3.954 & 5.968 & 4 & 2 & $12-5-66$ & $12-5-66$ \\
\hline OMNITAB (Ortho)...... & 1108 & GS & 4.137 & 5.464 & 2 & 4 & $10-18-67$ & $10-18-67$ \\
\hline ORTHO (no iteration).. & 1108 & GS & -1.976 & 0.419 & 20 & 20 & $3-7-68$ & $3-7-68$ \\
\hline ORTHOL $\ldots \ldots \ldots \ldots \ldots$ & 1108 & GS & 3.593 & 6.197 & 5 & 1 & $9-10-68$ & $9-10-68$ \\
\hline POLRG... & 1108 & E & -0.191 & 2.280 & 15 & 13 & $10-7-68$ & $10-7-68$ \\
\hline SPVMTX.. & 1108 & $\mathrm{E}$ & -0.658 & 1.527 & 18 & 17 & $11-14-67$ & $11-14-67$ \\
\hline STAT-PACK, GLH....... & 1108 & $\mathrm{E}$ & 0.066 & 2.767 & $10 \frac{1}{2}$ & $7 \frac{1}{2}$ & $11-7-67$ & $11-7-67$ \\
\hline STAT-PACK, REBSOM. & 1108 & $\mathrm{E}$ & 0.066 & 2.767 & $10 \frac{1}{2}$ & $7 \frac{1}{2}$ & $11-8-67$ & $11-9-67$ \\
\hline STAT-PACK, RESTEM. & 1108 & $\mathrm{E}$ & 0.651 & 2.407 & 9 & 10 & $7-2-68$ & $7-2-68$ \\
\hline WRAP.................... & 7094 & $\mathrm{E}$ & -5.300 & -2.871 & 22 & 22 & $6-28-67$ & $6-28-67$ \\
\hline
\end{tabular}

${ }^{\text {a }} \mathrm{E}=$ Elimination method; GS $=$ Gram-Schmidt orthonormalization; HT=Orthogonal Householder transformations; $\mathrm{OP}=$ Orthogonal polynomials.

TABLE 2. Summary of programs run in single precision - 9 digits

\begin{tabular}{|c|c|c|c|c|c|c|c|c|}
\hline \multirow{2}{*}{ Program } & \multirow{2}{*}{$\begin{array}{l}\text { Com- } \\
\text { puter }\end{array}$} & \multirow{2}{*}{$\begin{array}{c}\text { Algo- } \\
\text { rithm }\end{array}$} & \multicolumn{2}{|c|}{$\begin{array}{l}\text { Average number of } \\
\text { correct digits }\end{array}$} & \multicolumn{2}{|c|}{ Rank } & \multicolumn{2}{|c|}{ Date of run } \\
\hline & & & $Y 1$ & $Y 2$ & $Y 1$ & $Y 2$ & $Y 1$ & $Y 2$ \\
\hline LINFIT ***.. & 235 & $\mathrm{E}$ & 0.905 & 2.894 & 5 & 6 & $12-1-67$ & $12-1-67$ \\
\hline $\mathrm{LSCF}--* * *$. & 235 & $\mathrm{E}$ & 0.308 & 2.483 & 7 & 7 & $12-28-66$ & $12-28-66$ \\
\hline LSFITW $^{* * *} .$. & 235 & GS & 4.102 & 6.354 & 1 & 1 & $1-25-67$ & $1-25-67$ \\
\hline POLFIT ....... & 235 & OP & 3.349 & 5.922 & 2 & 2 & $2-19-68$ & $2-19-68$ \\
\hline SIMEX -***.. & 235 & $\mathrm{E}$ & 1.402 & 3.213 & 3 & 3 & $12-30-66$ & $1-5-67$ \\
\hline STAT $20 * * * .$. & 235 & $\mathrm{E}$ & 0.612 & 2.920 & 6 & 5 & $11-30-67$ & $11-30-67$ \\
\hline STAT $21^{* * *} \ldots \ldots \ldots$ & 235 & $\mathrm{E}$ & 1.169 & 3.183 & 4 & 4 & $1-3-67$ & $1-3-67$ \\
\hline
\end{tabular}

${ }^{\text {a }} \mathrm{E}=$ Elimination method; $\mathrm{GS}=\mathrm{Gram}-\mathrm{Schmidt}$ orthonormalization; OP=Orthogonal polynomials.

TABLE 3. Summary of programs run in double precision-16 digits

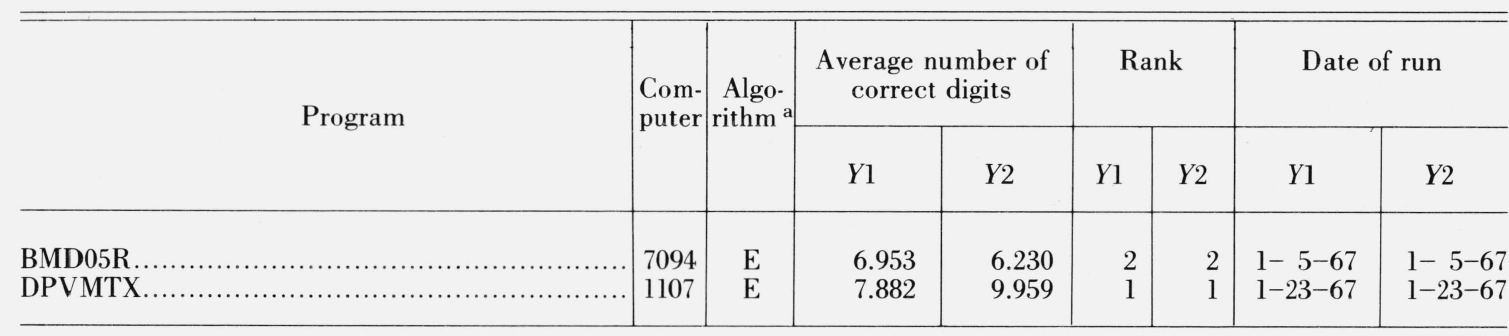

${ }^{\mathrm{a}} \mathrm{E}=$ Elimination method. 
TABLE 4. Summary of programs run in double precision-18 digits

\begin{tabular}{|c|c|c|c|c|c|c|c|c|}
\hline \multirow{2}{*}{ Program } & \multirow{2}{*}{$\begin{array}{l}\text { Com- } \\
\text { puter }\end{array}$} & \multirow{2}{*}{$\begin{array}{l}\text { Algo- } \\
\text { rithm a }\end{array}$} & \multicolumn{2}{|c|}{$\begin{array}{l}\text { Average number of } \\
\text { correct digits }\end{array}$} & \multicolumn{2}{|c|}{ Rank } & \multicolumn{2}{|c|}{ Date of run } \\
\hline & & & $Y 1$ & $Y 2$ & $Y 1$ & $Y 2$ & $Y 1$ & $Y 2$ \\
\hline ALSQ. & 1108 & HT & 12.667 & 15.322 & 4 & 4 & $10-19-67$ & $1-29-68$ \\
\hline BMD02R... & 1108 & $\mathrm{E}$ & 9.645 & 12.865 & 7 & 7 & $4-17-68$ & $4-17-68$ \\
\hline BMD05R... & 1108 & $\mathrm{E}$ & 9.368 & 11.791 & 9 & 10 & $9-10-68$ & $9-10-68$ \\
\hline DPVMTX. & 1108 & $\mathrm{E}$ & 9.744 & 13.484 & 6 & 6 & $2-27-68$ & $2-27-68$ \\
\hline LSTSQ ................... & 1108 & HT & 14.643 & 16.293 & 1 & 1 & $7-22-68$ & $7-22-68$ \\
\hline MATH-PACK, ORTHLS. & 1108 & $\mathrm{OP}$ & 12.098 & 14.461 & 5 & 5 & $10-16-68$ & $10-16-68$ \\
\hline ORTHO..................... & 1108 & GS & 13.188 & 15.514 & 3 & 3 & $1-29-68$ & $1-29-68$ \\
\hline ORTHO (no iteration)... & 1108 & GS & 7.963 & 10.354 & 11 & 11 & $3-7-68$ & $3-7-68$ \\
\hline ORTHOL $\ldots \ldots \ldots \ldots \ldots \ldots$ & 1108 & GS & 13.212 & 15.604 & 2 & 2 & $9-25-68$ & $9-25-68$ \\
\hline POLRG... & 1108 & $\mathrm{E}$ & 9.290 & 11.806 & 10 & 9 & $10-7-68$ & $10-7-68$ \\
\hline STAT-PACK, RESTEM.. & 1108 & $\mathrm{E}$ & 9.494 & 12.019 & 8 & 8 & $7-1-68$ & $7-1-68$ \\
\hline
\end{tabular}

${ }^{\text {a }} \mathrm{E}=$ Elimination method; GS=Gram-Schmidt orthonormalization; HT=Orthogonal Householder transformations; $\mathrm{OP}=$ Orthogonal polynomials.

TABlE 5. Summary of programs run in single precision (8 digits) with inner products accumulated in double precision (18 digits)

\begin{tabular}{|c|c|c|c|c|c|c|c|c|}
\hline \multirow{2}{*}{ Program } & \multirow{2}{*}{$\begin{array}{l}\text { Com- } \\
\text { puter }\end{array}$} & \multirow{2}{*}{$\begin{array}{l}\text { Algo- } \\
\text { rithm a }\end{array}$} & \multicolumn{2}{|c|}{$\begin{array}{l}\text { Average number of } \\
\text { correct digits }\end{array}$} & \multicolumn{2}{|c|}{ Rank } & \multicolumn{2}{|c|}{ Date of run } \\
\hline & & & $Y 1$ & $Y 2$ & $Y 1$ & $Y 2$ & $Y 1$ & $Y 2$ \\
\hline ALSQ.. & 1108 & HT & 3.506 & 6.530 & 3 & 1 & $10-18-68$ & $10-18-68$ \\
\hline LSTSQ... & 1108 & HT & 8.000 & 6.279 & 1 & 3 & $4-30-68$ & $5-6-68$ \\
\hline ORTHO... & 1108 & GS & 3.904 & 6.459 & 2 & 2 & $10-21-68$ & $10-21-68$ \\
\hline
\end{tabular}

${ }^{\text {a }} \mathrm{GS}=$ Gram-Schmidt orthonormalization; HT = Orthogonal Householder transformations.

TABLE 6. Summary of program run in multiple precision integer arithmetic

\begin{tabular}{|c|c|c|c|c|c|c|c|}
\hline \multirow{2}{*}{ Program } & \multirow{2}{*}{$\begin{array}{l}\text { Com- } \\
\text { puter }\end{array}$} & \multirow{2}{*}{$\begin{array}{l}\text { Algo- } \\
\text { rithm }^{\text {a }}\end{array}$} & & \multicolumn{2}{|c|}{$\begin{array}{l}\text { Average number } \\
\text { of correct digits }\end{array}$} & \multicolumn{2}{|c|}{ Date of run } \\
\hline & & & & Y1 & Y2 & Y1 & Y2 \\
\hline SOLVER... & 1108 & C & $\begin{array}{l}\text { Rational form } \\
\text { Floated form }\end{array}$ & $\stackrel{\infty}{\infty} 18.000$ & $\begin{array}{c}\infty \\
17.347\end{array}$ & $7-16-68$ & $7-16-68$ \\
\hline
\end{tabular}

${ }^{\text {a }} \mathrm{C}=$ Congruential method.

For two programs reported in table 1, BMD03R run on the 7094 and DAM run on the 7094, the count for several coefficients was made in a different manner. The BMD03R program printed the coefficients in a fixed-decimal format, with five decimals. The DAM program used a floatingpoint format with only three decimals printed. A coefficient printed as 0.00010 , when the true coefficient was 0.0001 , was given a count of 2 , and $0.100 \mathrm{E} 01$, when the true coefficient was 1 ., was given a count of 3 . In such cases the assigned count may have been too small, since the coefficients may have been calculated accurately to more digits than were printed. In running these two programs on the 1108, the output format was changed so that eight significant digits were printed. 
Each of the tables (1 through 6) summarizes a set of results for a particular machine precision $-8,9,16,18$, etc. digits. Within each table the various programs are ranked for each of the two test problems, with rank 1 denoting the best performance according to the count $\mathrm{C}$.

Table 1 includes single precision (8-digit) programs run on two different computers, the 7094 and the 1108. It was felt that combining the results from two computers was justified in view of the similar performance of the four programs which were run on both computers. These four programs were BMD03R, DAM, OMNITAB (using INVERT), and OMNITAB (using ORTHO). The average number of correct digits for the two test problems from the 1108 agrees with the corresponding average from the 7094 to within 0.9 digits except in the case of $Y 1$ run on DAM, where the difference is 1.653. This larger difference may possibly be attributable to modifications in the program. Furthermore, other test problems have been run on OMNITAB (using ORTHO) on both computers, and again the results were quite similar.

The symbols in the Algorithm column of the tables denote the following:

C Congruential method, integer arithmetic

E Elimination method

GS Gram-Schmidt orthonormalization

HT Orthogonal Householder transformations

OP Orthogonal polynomials.

From time to time at a given computer installation changes are made in hardware and software with the result that a particular job run on two different days may not produce identical numerical output. For this reason the date of the computer runs is included in the tables.

Details (individual coefficients and counts) supporting tables 1 through 6 are given in appendix B.

\section{Programs Using Orthogonal Householder Transformations}

LSTSQ is a program written by Peter A. Businger using orthogonal Householder transformations. This algorithm is described by Golub [21] and Businger and Golub [8]. The program applies a sequence of orthogonal transformations to the $n$ by $k$ least squares matrix $X$ to obtain a decomposition $X=Q R$, where $R$ is upper triangular and $Q^{\prime} Q=I_{k}$. A pivoting strategy is used so that at each step the column with the largest sum of squares is reduced next. Once an initial solution is obtained, the program iterates to obtain a (possibly) improved solution.

Of all the programs using floating-point arithmetic included in this study, LSTSQ appears to have given the best performance. In table 4, which reports the performance of eleven double precision programs, we see that LSTSQ ranked first for both $Y 1$ and $Y 2$. In table 1, which reports the performance of 22 single precision programs, we see that LSTSQ obtained rank 1 for $Y 1$ and rank 3 for $Y 2$. Ranks 1 and 2 for the $Y 2$ problem were obtained by ORTHOL and OMNITAB (using ORTHO), two programs using Gram-Schmidt orthonormalization which will be discussed more fully in the next section. Table 5 reports the performance of three programs which used single precision arithmetic except for the accumulation of inner products, where double precision arithmetic was used. Here we see that LSTSQ ranked first for $Y 1$ (having a perfect score of 8.000) and ranked third for Y2. In the two instances just mentioned where LSTSQ ranked third for $Y 2$, we see that the difference separating it from the top-ranking program is small, being 0.357 in table 1 and 0.251 in table 5 .

Golub and Businger recommend that all inner products be accumulated in double precision. By comparing tables 5 and 1 we see that when LSTSQ included this feature, the average counts increased from 4.528 to 8.000 for $Y 1$ and from 5.840 to 6.279 for $Y 2$. With all operations performed in double precision (see table 4), the counts increased to 14.643 and 16.293 , respectively.

The other program using Householder transformations was ALSQ, written by G. W. Stewart, III. This program contains no pivoting and no iteration. In tables 1,4 , and 5 we see that ALSQ performed not quite as well as LSTSQ which included these features, except in one instance. In this one instance, $Y 2$ in table 5, we note that its performance was slightly better than that of LSTSQ. 
By examining tables 1 and 5, one may see the effect of accumulating inner products in double precision versus accumulating them in single precision. As one would expect, ALSQ did better in computing the coefficients of $Y 2$ when the double precision accumulation was included. For $Y 1$, surprisingly, we see that ALSQ lost accuracy with this feature included. A look at the details of the program revealed how this phenomenon occurred. After the matrix $X$ has been decomposed to obtain $Q R$ (as described earlier), the coefficients are computed by back-substitution. The first coefficient to be computed is $\hat{\beta}_{6}$ (the coefficient for the fifth-degree term), and this is obtained from one arithmetic operation, a division. Correctly calculated to ten digits, this division is $\frac{21011.77901}{21011.77901}=1$. In the single precision version, the coefficient was calculated as

$$
\hat{\beta}_{6}=\frac{21011.714}{21011.713}=1.0000000(\text { to } 8 \text { significant digits })
$$

whereas in the version with inner products accumulated in double precision the calculation was

$$
\hat{\beta}_{6}=\frac{21011.761}{21011.753}=1.0000004(\text { to } 8 \text { significant digits }) .
$$

We note that in (1), both the numerator and denominator in question are farther from their true values than in (2), but they are closer to each other, so that $\hat{\beta}_{6}$ in (1) happens to be closer to the true value of $\hat{\beta}_{6}$. Subsequently, $\hat{\beta}_{6}$ enters into the calculations of the five other coefficients with the result that all the coefficients for $Y 1$ from the single precision version are slightly more accurate than those from the version using double precision inner products.

\section{Programs Using Gram-Schmidt Orthonormalization}

ORTHO is a program written by Philip J. Walsh using a Gram-Schmidt orthonormalization process. This algorithm is described by Davis and Rabinowitz [13], [14], Davis [12], and Walsh [42]. ORTHO exists as a FORTRAN program, an ALGOL procedure, a BASIC program, and as a routine of the OMNITAB program (see Hilsenrath, et al., [23]), where it is called by the commands FIT and POLYFIT.

Starting with the $n \times k$ matrix $X$, the Gram-Schmidt process of ORTHO obtains $\varphi=X T^{\prime-1}$ and $\hat{\beta}=T^{\prime-1} \varphi^{\prime} Y$, where $T^{\prime-1}$ is upper triangular and $\varphi^{\prime} \varphi=I_{k}$. This algorithm includes a feature of reorthonormalizing the vectors of $\varphi$, proceeding from a first approximation $\bar{\varphi}_{j}$ to a (usually) better approximation $\varphi_{j}$. From table 1 it is clear that this reorthonormalizing is vital to the algorithm, for ORTHO's good performance in handling $Y 1$ and $Y 2$ deteriorated when this iteration was omitted. For $Y 1$, the count of correct digits dropped from 4.137 to -1.976 , and for $Y 2$ the drop was from 5.464 to 0.419 . In table 4 , also, we see that in double precision the omission of the iteration resulted in a loss of about five correct digits for both problems.

The LSFITW*** program, written in BASIC, listed in table 2 also uses the ORTHO algorithm. The computer used for running the programs of table 2 works with about one more decimal digit than the computers covered in table 1 , so one would expect more accuracy from LSFITW*** than from OMNITAB (ORTHO). We find slight improvement for $Y 2$, but no improvement for $Y 1$. Of the seven programs reported in table 2, LSFITW ${ }^{* * *}$ ranked 1 on both problems. Note that there are no Householder transformation programs included in table 2.

The ORTHO program was also run in a version using single precision except for the accumulation of inner products, where double precision was used. In table 5 we see that there were three programs in this category, and for both problems ORTHO ranked second. Its performance on $Y 2$ improved by about one digit compared to the performance of the ORTHO version using strictly single precision. On the $Y 1$ problem, however, there was a slight loss in accuracy. Actually, three coefficients gained accuracy and three lost accuracy, with a net loss in the average count. (A similar loss which occurred with ALSQ was discussed in the previous section.) In ORTHO, the 
final calculation to obtain the coefficients $\hat{\beta}$ is the matrix multiplication $\hat{\beta}=\left(T^{\prime}\right)^{-1} \alpha$, where $\left(T^{\prime}\right)^{-1}$ is an upper triangular matrix such that $\left(T^{\prime}\right)^{-1} T^{-1}=\left(X^{\prime} X\right)^{-1}$, and $\alpha=T^{-1} X^{\prime} Y$. Nearly all of the nonzero elements of $\left(T^{\prime}\right)^{-1}$ and $\alpha$ were more accurately computed when inner products were accumulated in double precision than when this feature was omitted. In the three coefficients of $Y 1$ which lost accuracy, an examination of the details showed that in the individual multiplications involved in the matrix multiplication, the version using double precision for inner products was always more accurate than the strictly single precision version. But in the final addition of the various products, where the terms have alternating signs, there was heavy cancellation and the errors combined in such a way that the $\hat{\beta}$ 's from the single precision version happened to be closer to the true values of the coefficients than those computed with double precision accumulation of inner products.

ORTHOL is a program using a modification of the Davis-Rabinowitz algorithm written by James W. Longley and Roger A. Blau [30]. It differs from Walsh's ORTHO in two respects: (1) the iteration procedure includes the dependent variable as well as the independent variables, and (2) before any other operations are applied to the matrix $X$, from each element of each vector of $X$. the truncated mean of that vector is subtracted. (The "truncated mean" denotes the largest integer less than or equal to the mean if the mean is nonnegative, and the smallest integer greater than or equal to the mean if the mean is negative.) ORTHOL obtained the top rank for $Y 2$ in single precision, but ranked fifth for $Y 1$ (table 1). In double precision (table 4), it ranked second on both problems.

\section{Programs Using Orthogonal Polynomials}

Since the two test problems are both polynomial fits, we were able to test programs in which the algorithm used orthogonal polynomials. This method, described by Forsythe [18], is attractive because it generally requires many fewer operations than other methods.

Two such programs were included in this study. One was the UNIVAC 1108 MATH-PACK routine, ORTHLS (see Programmers Reference [40]). The other was POLFIT, an anonymous program written in BASIC.

In tables 1, 2, and 4 we see that the performance of the orthogonal polynomial programs is not as good as that of the Householder transformation and the Gram-Schmidt programs (with iteration), but the performance is better than that of any of the programs using elimination algorithms. This finding is in agreement with the results of Bright and Dawkins [7] who ran a number of polynomial test problems via two methods: matrix inversion using a Gauss-Jordan reduction, and orthogonal polynomials. In all cases they found the orthogonal polynomial method superior.

\section{A Multiple Precision Integer Arithmetic Program Using Congruential Methods}

Morris Newman, in his paper "Solving Equations Exactly" [35] described a congruential method for finding the exact solution of a system of linear equations $A x=b$ where the elements of $A$ and $b$ are all integers. His FORTRAN program SOLVER will solve systems in which $A$ is a square matrix at most 100 by 100 and the elements of $A$ and $b$ are numerically less than $10^{20}$. This method is not at all sensitive to the condition of $A$, but it can be time-consuming for large systems. ${ }^{2}$ The solution is printed in two versions: (1) $x=\left(\frac{1}{\operatorname{det} A}\right) z$, where $z$ is a vector of integers and the determinant $\operatorname{det} A$ is an integer, and (2) $x$ in floated double-precision format, accurate to about 17 digits on the 1108

The two test problems, $Y 1$ and $Y 2$, were run on this program, as indicated in table 6 . The input required was the matrices $X^{\prime} X$ and $X^{\prime} Y$. Since the elements of $X^{\prime} Y$ for $Y 2$ are not integers, it was necessary to multiply these numbers by 100,000 before obtaining the solution.

\footnotetext{
2 The running time on the Univac 1108 for the solution of $Y 1$ and $Y 2$ was 11 seconds, including 5 seconds for compilation of the program. The six problems de scribed in the latter part of this section, all having $6 \times 6$ systems, required 14 seconds, including 4 seconds for compilation. To solve a $20 \times 20$ system, the worst possible case requires about 30 seconds, and an "average" case takes less time. For a $40 \times 40$ system, an average case requires about one minute, and the worst possible case requires about six minutes. A "bad" $100 \times 100$ case might require 40 minutes or more running time.
} 
Having a program which produces exact solutions, we can determine what will happen to the solution when we round the input, the elements of $X^{\prime} X$ and $X^{\prime} Y$. These elements for $Y 1$ are integers having no more than 14 digits. Six additional problems were run in which the input was successively rounded to $13,12,11,10,9$, and 8 significant digits. The effect of this rounding was to change the solution dramatically. In table 7 , which gives the solutions to these six problems (rounded to 10 decimals), we see that "small" changes to the elements of $X^{\prime} X$ and $X^{\prime} Y$ produce "large" changes to the solution, $\hat{\beta}$.

At first glance, the fact that the coefficients calculated for the problem having input rounded to 13 significant digits agree with the coefficients obtained from unrounded input to only $4,5,6$, or 8 digits seems quite surprising. But with a few simple calculations we can see why the agreement is no better than it is. First, referring to table 9, we note that there is only one 14-digit number in $X^{\prime} X$, and since this ends with a zero, rounding to 13 significant digits leaves $X^{\prime} X$ unaltered. In $X^{\prime} Y$ only the last element has 14 digits. Here, 25,537,373,767,266 was rounded to 25,537,373,767,270. Let $B=\left(b_{i j}\right), i, j=1, \ldots, 6$, denote the inverse of $X^{\prime} X$ and let $\left(q_{j}\right)=X^{\prime} Y, j=1, \ldots, 6$. Consider $\hat{\beta}_{1}$, the first coefficient. We have

$$
\hat{\beta}_{1}=b_{11} q_{1}+b_{12} q_{2}+b_{13} q_{3}+b_{14} q_{4}+b_{15} q_{5}+b_{16} q_{6}=\sum_{j=1}^{5} b_{1 j} q_{j}+b_{16} q_{6}
$$

The only quantity which is affected by changing from unrounded data to rounded data with 13 significant digits is $q_{6}$. Now

$b_{16}=-28,046,715,376,452,025,326,796,800 /\left(\operatorname{Det} X^{\prime} X\right)$, where

Det $X^{\prime} X=1,677,193,579,511,831,114,542,448,640,000$. Since $q_{6}=25,537,373,767,266$ we have

$$
b_{16} q_{6}=-716,239,453,512,581,907,404,696,441,196,473,548,800 /\left(\operatorname{Det} X^{\prime} X\right) .
$$

Also,

$$
\sum_{j=1}^{5} b_{1 j} q_{j}=716,239,455,189,775,486,916,527,555,738,922,188,800 /\left(\operatorname{Det} X^{\prime} X\right) .
$$

In combining the last two numbers, one positive and the other negative, we see that the first eight digits of the two numerators are identical, so that the numerator of $\hat{\beta}_{1}$ has eight fewer digits than has $\sum_{j=1}^{5} b_{1 j} q_{j}$ or $b_{16} q_{6}$.

In solving for $\hat{\beta}_{1}^{\prime}$ from input rounded to 13 significant digits, we have $q_{6}^{\prime}=25,537,373,767,270$ so that

$$
b_{16} q_{6}^{\prime}=-716,239,453,512,694,094,266,202,249,297,780,736,000 /\left(\operatorname{Det} X^{\prime} X\right) \text {. }
$$

The numerator here differs from the numerator of $b_{16} q_{6}$ in the thirteenth significant digit, but after combining this with $\sum_{j=1}^{5} b_{1 j} q_{j}$ and losing eight significant digits, we obtain

$$
\begin{aligned}
\hat{\beta}_{1}^{\prime} & =\sum_{j=1}^{5} b_{1 j} q_{j}+b_{16} q_{6}^{\prime} \\
& =\frac{1,677,081,392,650,325,306,441,141,452,800}{1,677,193,579,511,831,114,542,448,640,000} \\
& =0.9999331104 \text { (to } 10 \text { decimals). }
\end{aligned}
$$

There are similar losses of significant digits in calculating the other coefficients.

A rigorous presentation of the sensitivity of the solution of a system of equations $A x=b$ with respect to variations in $A$ and $b$ is given in Wilkinson [43, p. 91] and Wilkinson [44, p. 189]. 
TABLE 7. Exact solutions to approximate problems

The column $\mathrm{A}(\mathrm{N})$ gives a count of the number of digits in the solution of $\left(X^{\prime} X\right) \beta=X^{\prime} Y$ for input rounded to $N$ digits which are in agreement with the solution for un rounded input. The unrounded input (elements of $X^{\prime} X$ and $X^{\prime} Y$ ) consisted on integers having no more than 14 digits.

\begin{tabular}{|c|c|c|c|c|}
\hline $\begin{array}{l}\text { Solution for unrounded } \\
\text { input }\end{array}$ & $\begin{array}{l}\text { Solution for input } \\
\text { rounded to } 13 \text { sig. digits }\end{array}$ & $\mathrm{A}(13)$ & $\begin{array}{l}\text { Solution for input } \\
\text { rounded to } 12 \text { sig. digits }\end{array}$ & $\mathrm{A}(12)$ \\
\hline \multirow[t]{5}{*}{$\begin{array}{l}1 . \\
1 . \\
1 . \\
1 . \\
1 . \\
1 .\end{array}$} & $\begin{array}{ll}0.99993 & 31104 \\
1.00015 & 06443 \\
0.99994 & 21662 \\
1.00000 & 79679 \\
0.99999 & 95470 \\
1.00000 & 00091\end{array}$ & $\begin{array}{r}4.175 \\
3.822 \\
4.238 \\
5.099 \\
6.344 \\
8.043 \\
87\end{array}$ & $\begin{array}{ll}0.99672 & 54481 \\
1.00718 & 28792 \\
0.99727 & 93936 \\
1.00037 & 12813 \\
0.99997 & 90427 \\
1.00000 & 04168\end{array}$ & $\begin{array}{r}2.485 \\
2.144 \\
2.565 \\
3.430 \\
4.679 \\
6.380 \\
614\end{array}$ \\
\hline & $\begin{array}{l}\text { Solution for input } \\
\text { rounded to } 11 \text { sig. digits }\end{array}$ & $\mathrm{A}(11)$ & $\begin{array}{l}\text { Solution for input } \\
\text { rounded to } 10 \text { sig. digits }\end{array}$ & $\mathrm{A}(10)$ \\
\hline & $\begin{array}{lll}1.06051 & 32874 & \\
0.86927 & 95602 & \\
1.04911 & 27057 & \\
0.99333 & 63623 & \\
1.00037 & 44589 & \\
0.99999 & 25798 & \\
& & \text { Avg }\end{array}$ & $\begin{array}{r}1.218 \\
0.884 \\
1.309 \\
2.176 \\
3.427 \\
5.130 \\
57\end{array}$ & $\begin{array}{ll}0.91988 & 69708 \\
1.19460 & 48731 \\
0.92297 & 46106 \\
1.01080 & 85953 \\
0.99937 & 78148 \\
1.00001 & 25555\end{array}$ & $\begin{array}{r}1.096 \\
0.711 \\
1.113 \\
1.966 \\
3.206 \\
4.901 \\
66\end{array}$ \\
\hline & $\begin{array}{l}\text { Solution for input } \\
\text { rounded to } 9 \text { sig. digits }\end{array}$ & $\mathrm{A}(9)$ & $\begin{array}{l}\text { Solution for input } \\
\text { rounded to } 8 \text { sig. digits }\end{array}$ & $\mathrm{A}(8)$ \\
\hline & $\begin{array}{rr}3.70810 & 09327 \\
-4.34362 & 06781 \\
2.92257 & 14823 \\
0.74656 & 29295 \\
1.01394 & 46989 \\
0.99972 & 81121\end{array}$ & $\begin{array}{r}-0.433 \\
-0.728 \\
-0.284 \\
0.596 \\
1.856 \\
3.566 \\
62\end{array}$ & $\begin{array}{rr}-24.56199 & 35653 \\
52.60541 & 27451 \\
-17.89853 & 20445 \\
3.52648 & 11096 \\
0.85941 & 47174 \\
1.00276 & 62377\end{array}$ & $\begin{array}{l}-1.408 \\
-1.713 \\
-1.276 \\
-0.403 \\
0.852 \\
2.558 \\
.232\end{array}$ \\
\hline
\end{tabular}

\section{Programs Using Elimination Algorithms}

The majority of the programs tested in this investigation used some form of an elimination algorithm. Although this was the most popular method, it was the least successful. None of these programs performed as well as those using Householder's transformations, Gram-Schmidt orthonormalization (with iteration), or orthogonal polynomials.

Within this class of programs, there were several variations in the method of obtaining the least-squares coefficients. In some cases, the matrix $X^{\prime} X$ was inverted, after which the inverse was postmultiplied by $X^{\prime} Y$ to obtain $\hat{\beta}=\left(X^{\prime} X\right)^{-1} X^{\prime} Y$. In one program the matrix

$A=\left[\begin{array}{cr}X^{\prime} X & X^{\prime} Y \\ 0 & 1\end{array}\right]$ was inverted. Here, the inverse is

$A^{-1}=\left[\begin{array}{cc}\left(X^{\prime} X\right)^{-1} & -\hat{\beta} \\ 0 & 1\end{array}\right]$. Another program inverted the matrix $Z^{\prime} Z$

where the vectors of $Z$ were obtained from the vectors of $X$ by subtracting the mean of each vector from every element of that vector. A number of programs obtained the solution by inverting a matrix of correlation coefficients. The five stepwise regression programs made use of matrix partitioning in connection with inverting a matrix of correlation coefficients.

\subsection{Stepwise Regression Programs}

The five stepwise regression programs were BMD02R, MPR3, the STAT-PACK program RESTEM, WRAP, and STAT20***. They all, to a greater or lesser extent, follow Efroymson's algorithm [16]. Tables 1, 2 and 4 give the results of these five programs. 
The BMD02R program is described in BMD Biomedical Computer Programs [15]. The UNIVAC 1108 STAT-PACK Program RESTEM is described in the Programmers Reference [41]. The two programs, MPR3 and WRAP, are both from the SHARE library [26]. The former was written by M. A. Efroymson and the latter was written by M. D. Fimple. The program STAT20*** is included in the C-E-I-R Multi-Access Computer Service library, and a brief writeup on how to use the program is given in C-E-I-R's "Library Programs Documentation" [10].

In running the two test problems on three of the stepwise programs, namely BMD02R, RESTEM and STAT20***, calculations stopped before the solutions were obtained. In all three programs, computations stopped in the $Y 2$ problem after $x, x^{2}, x^{4}, x^{5}$ and a constant term had entered the regression equation. In the $Y 1$ problem, BMD02R and STAT20*** stopped after $x^{4}, x^{5}$ and a constant term had entered, and RESTEM stopped after $x^{5}$ and a constant term had entered. In order to obtain the coefficients for the fifth degree equations, certain features of these three programs had to be bypassed.

In the printout of RESTEM and STAT20*** obtained from the original (unaltered) programs, there were no clues to indicate that there was a rounding error problem. The initial runs of the BMD02R program, however, printed the messages "ERROR TERMINATION IN SQRT ROU. TINE" and "SQRT CALLED AT SEQUENCE NUMBER 01032 OF MAIN PROGRAM." These messages were produced by the computer system, not by the BMD02R program. They indicated the nature of some of the trouble-that the argument of a certain square root function was negative. The initial BMD02R runs furnished another clue of computational difficulties. The output of this program includes various calculated $F$-values which are needed for entering and removing variables from the regression. In both $Y 1$ and $Y 2$, there were one or more $F$-values (labeled " $F$ TO ENTER") which were negative.

It was found that the RESTEM and STAT20*** programs had also calculated negative $F$. values, and checks involving $F$-values had to be bypassed in order to obtain the fifth degree solutions. Moreover, in the RESTEM program it was necessary to change the value of "minus infinity" from $-10^{38}$ to $-10^{34}$ before satisfactory results could be obtained for any least squares problem.

WRAP, the program with the lowest rankings in table 1, computed coefficients which were exceptionally far from the true values. These coefficients are listed below.

\begin{tabular}{c|c|c|c}
\hline \multicolumn{2}{c|}{$Y 1$} & \multicolumn{2}{|c}{$Y 2$} \\
\hline True $\hat{\beta}$ & Computed $\hat{\beta}$ & True $\hat{\beta}$ & Computed $\hat{\beta}$ \\
\hline & & & -33.84546 \\
1. & $29 \overline{9} 1622$. & 1. & 71.54880 \\
1. & -6065892. & .1 & -01 \\
1. & 2218821. & .001 & 3.493256 \\
1. & -296194.5 & .0001 & -0.1936966 \\
1. & 16462.20 & .00001 & .003812985 \\
1. & -322.5731 & & \\
\hline
\end{tabular}

Since WRAP performed so poorly on the two test problems, $Y 1$ and $Y 2$, some other test problems were run in order to verify that the program could handle problems which were not so badly conditioned. Let $U 1(k)$ and $U 2(k)$ be defined as follows:

$$
\begin{aligned}
& U 1(k): y=1+x+x^{2}+\ldots+x^{k}, \\
& U 2(k): y=1+0.1 x+0.01 x^{2}+\ldots+10^{-k} x^{k} .
\end{aligned}
$$

Taking $x=0(1) 20, k=1,2,3,4$, the $y$-values were calculated for $U 1(k)$ and $U 2(k)$. Using these calculated $y$ 's as input, it was found that the coefficients for degrees 1,2 , and 3 computed by the WRAP 
program had some accuracy, but those for degree 4 were computed inaccurately. The results for degrees 3 and 4 are given below.

\begin{tabular}{c|c|c|c}
\hline \multicolumn{2}{c|}{$U 1(3)$} & \multicolumn{2}{c}{$U 2(3)$} \\
\hline True $\hat{\beta}$ & Computed $\hat{\beta}$ & True $\hat{\beta}$ & Computed $\hat{\beta}$ \\
\hline & & & \\
1. & 1.223297 & 1. & 1.000416 \\
1. & 0.8286224 & 0.1 & 0.09971083 \\
1. & 1.022150 & .01 & .01003707 \\
1. & 0.9992682 & .001 & .0009987662 \\
\hline
\end{tabular}

\begin{tabular}{c|c|c|c}
\hline \multicolumn{2}{c|}{$U 1(4)$} & \multicolumn{2}{c}{$U 2(4)$} \\
\hline True $\hat{\beta}$ & Computed $\hat{\beta}$ & True $\hat{\beta}$ & Computed $\hat{\beta}$ \\
\hline & & & \\
1. & -731.1589 & 1. & .8673919 \\
1. & 852.1714 & 0.1 & .2555575 \\
1. & -193.1382 & .01 & .02548887 \\
1. & 15.94353 & .001 & .003731290 \\
1. & 0.6330207 & .0001 & .00003291620 \\
\hline
\end{tabular}

\subsection{Other Programs Using Elimination Algorithms}

Two other BMD programs were tested. The BMD03R program, Multiple Regression with Case Combinations, inverts a matrix of correlation coefficients. BMD05R, Polynomial Regression, inverts the matrix $Z^{\prime} Z$ where the vectors of $Z$ are formed from the vectors of $X$ by subtracting the mean of each vector from every element of that vector. All the crucial operations of BMD05R, such as the forming of inner products and matrix inversion, are carried out in double precision. The performance of BMD03R and BMD05R is shown in tables 1,3 , and 4.

DAM is a general-purpose computer program for data processing and multiple regression written by Rudolf R. Rhomberg, Lorette Boissonneault, and Leonard Harris, International Monetary Fund [36]. In running the two test problems on DAM on the 1108, computations stopped after a fourth degree polynomial was fitted, and the message "INSUFFICIENT NUMBER OF OBSERVATIONS OR DATA ARE ALL ZEROS, PROGRAM CANNOT COMPUTE EQUATION 5" was printed. It was found that a computed variance was zero and that this condition causes the computations to stop. By bypassing the checks on this computed variance, results for fifth degree fits were obtained. On the 7094, however, the fifth degree results were reached without any such difficulties. DAM's performance on the two computers is given in table 1 .

Two lines of table 1 report the results from OMNITAB on the 7094 and the 1108 where the matrix commands INVERT, MMULT, and MTRANS were used. Here the 21 pairs of $(x, y)$ values were read into the computer, the powers of $x$ were generated, the matrices $X^{\prime} X$ and $X^{\prime} Y$ were obtained via MTRANS and MMULT, the inverse of $X^{\prime} X$ was obtained via INVERT, and $\hat{\beta}$ was then obtained via MMULT. The solutions were far less accurate than those obtained from OMNITAB by using the command POLYFIT which calls on the ORTHO routine.

The program POLRG is the polynomial regression program of the IBM System/360 Scientific Subroutine Package [24], [25]. This program calls four subroutines, GDATA, ORDER, MINV, and MULTR, in the course of obtaining the least squares coefficients and other quantities of interest. These subroutines perform the following operations:

(1) GDATA generates the powers of the independent variable, finds means and standard deviations, and sets up a correlation matrix. 
(2) ORDER chooses a dependent variable and a subset of independent variables from a larger set of variables.

(3) MINV inverts the correlation matrix using the "standard Gauss-Jordan method."

(4) MULTR computes the regression coefficients and related quantities, such as the sum of squares attributable to the regression and the sum of squares of deviations from the regression.

We see from table 1 that the single precision version of POLRG obtained rather low scores on both test problems. A double precision version of POLRG was also run, and the performance here as reported in table 4 was comparable to other programs using similar elimination algorithms.

The user of POLRG specifies $m$, the highest degree polynomial to be fitted, and the program automatically reports the results of fitting polynomials of successively increasing degrees, starting with the first degree. If there is no reduction in the residual sum of squares between two successive degrees of polynomials, the program stops the problem before completing the analysis for the highest degree specified. In running both test problems, $Y 1$ and $Y 2$, in single precision the analysis stopped after degree four, and in lieu of a fifth degree polynomial fit, the message "NO IMPROVEMENT" was printed. In order to complete the calculations for the fifth degree, the checks on "improvement" were bypassed. In the double precision version, fifth degree results were obtained without any such alterations.

The Programmer's Manual for the IBM System/360 Scientific Subroutine Package [25] contains some warnings regarding the accuracy of computations. The reader is informed that the accuracy of the computations in many of the routines is highly dependent upon the number of significant digits available for arithmetic operations. It is pointed out that matrix inversion and many of the statistical subroutines fall into this category, and that the user may, therefore, wish to use double precision versions of these routines. (The programs are so constructed that conversion to double precision is an easy matter.) An appendix of the manual classifies the subroutines of this package into three categories. These are: (1) subroutines having little or no effect on accuracy, (2) subroutines whose accuracy is dependent on the characteristics of the input data, and (3) subroutines in which definite statements on accuracy can be made. Only one of the four subroutines called by the POLRG program, namely ORDER, is in the first category. The other three subroutines, GDATA, MINV and MULTR, fall in the second category. In connection with this second category we read that "it cannot be assumed that the results are accurate simply because subroutine execution is completed."

A more explicit statement is given in connection with the subroutine GDATA. Here there is a comment in the program stating that if $m$, the highest degree polynomial to be fitted, is equal to 5 or greater, single precision may not be sufficient to give satisfactory results. Since the manual's test problem for POLRG specifies $m=4$ and has 15 data points, one might infer that satisfactory results would be obtained for this problem. This is not the case, however. In the solution to this problem given on page 410 of the manual, the intercept term for the polynomial regression of degree 4 is reported to be -5.26735 . An accurate calculation shows that this term is actually -6.04262 , so that the reported term had no correct significant digits. The four reported regression coefficients were correctly computed to only one or two digits. Furthermore, the sum of squares of deviations from the regression is reported to be 128.85156, whereas it is actually 17.67310. This error is also propagated into the calculation of the mean square, the $F$ value, and the improvement in terms of sum of squares. The calculated values of $\hat{Y}$ were found to be correct to one, two or three significant digits, with the residuals correct to one digit or less.

In concluding this digression concerning the accuracy of the test problem accompanying a particular program of a particular package, we note a remark given in the Programmer's Manual under "Purposes and Objectives of the Package": "While this package may provide many of the tools necessary to solve the more commonly encountered problems in engineering and science, there is no intent to imply that these subroutines represent the current state of the art in statistics or numerical analysis."

The programs SPVMTX and DPVMTX appearing in tables 1, 3, and 4 use single and double precision versions, respectively, of the same algorithm. These two programs were adapted by 
Sally T. Peavy, National Bureau of Standards, from two subroutines in the SHARE library: A. R. Sadaka's 7090-F1 3180INV1 Single Precision Matrix Inversion with Selective Pivoting and A. R. Sadaka's 7090-F1 3181INV2 Double Precision Matrix Inversion with Selective Pivot. Mrs. Peavy's adaptations of these programs included accuracy checks on the computed inverse. A brief description of these accaracy checks is in order. Let $A$ be the input matrix to be inverted and $Z$ the result of the inversion. Let $E=I-A Z$. Let $B=\left(b_{i j}\right)$ be an $n \times n$ matrix, and let $N(B)$ be a norm defined in any of the following ways:

$$
\begin{aligned}
& N_{1}(B)=\left(\sum_{i, j}\left|b_{i j}\right|^{2}\right)^{1 / 2} \text { (the Euclidean or Frobenius norm) } \\
& N_{2}(B)=n \max _{i, j}\left|b_{i j}\right| \\
& N_{3}(B)=\max _{i} \sum_{j=1}^{n}\left|b_{i j}\right| .
\end{aligned}
$$

In order to guarantee that $Z$ be a good approximation to $A^{-1}$, it is only necessary to have $\frac{N(Z) N(E)}{1-N(E)}$ small. This quantity, $\frac{N(Z) N(E)}{1-N(E)}$, is computed for each of the three norms $N_{1}, N_{2}$, and $N_{3}$, and provides upper bounds on the error in the elements of the computed inverse. See Newman [34, pp. 227-230] and Taussky [38, pp. 284-286] for a fuller discussion of the norms described above.

The matrix which was inverted by SPVMTX and DPVMTX in solving the two test problems was

$$
\left[\begin{array}{cc}
X^{\prime} X & X^{\prime} Y \\
0 & 1
\end{array}\right] \text { whose inverse is }\left[\begin{array}{cc}
\left(X^{\prime} X\right)^{-1} & -\hat{\beta} \\
0 & 1
\end{array}\right] \text {. }
$$

In the single precision solution, the three bounds for $Y 1$ were, respectively, $-160,-940$, and -140 , and those for $Y 2$ were $-2.2,-7.0$ and -2.7 . If an accurate inverse had been obtained, the error bounds would have been small positive numbers. That the bounds were negative is a clear warning that the computed inverses, including $\hat{\beta}$, are not accurate. The double precision 1108 solution obtained the bounds $0.00048,0.0075$, and 0.00046 for $Y 1$, and $0.000000039,0.00000064$, and 0.000000087 for $Y 2$. In both problems these bounds are quite conservative. In the solution of $Y 1$, the largest error in the elements of $\left(X^{\prime} X\right)^{-1}$ is $5.5 \times 10^{-13}$, and the largest error in the $\hat{\beta}_{j}$ s is $5.5 \times 10^{-7}$. In the solution of $Y 2$, the largest error in the elements of $\left(X^{\prime} X\right)^{-1}$ is again $5.5 \times 10^{-13}$, and in the $\hat{\beta}_{j}$ 's is $3.3 \times 10^{-13}$.

The 1108 version of OMNITAB now uses the SPVMTX routine for matrix inversion and prints out the smallest error bound. OMNITAB reported the smallest error bound for the inverse of $X^{\prime} X$ to be -6 , a negative number. This was in agreement with the results from inverting $X^{\prime} X$ via the FORTRAN program SPVMTX where the three error bounds were given as $-1.7,-6.0$ and -2.1 .

Each of the two STAT-PACK programs, GLH, Geñeral Linear Hypotheses, and REBSOM, Back Solution Multiple Regression, has its individual features, but for the two test problems the solutions were carried out in the same manner, so that the coefficients obtained from the two programs were identical, as is indicated in table 1 . Both programs invert $X^{\prime} X$ by calling a matrix inversion subroutine called JIM which uses a Gauss-Jordan elimination scheme with maximal column pivoting and row scaling. The GLH program has an option whereby the user can enter restraints in the case $X^{\prime} X$ is not of full rank. The REBSOM program has the feature that the user can enter an $F$-value to be used as a criterion for removing variables from the regression after an initial solution has been computed.

An error was found in the REBSOM program in the calculation of the variance of $Y$. After estimating $k$ coefficients (including possibly a constant term) from $n$ observations, the formula used for the variance of $Y$ is var $Y=\frac{\sum\left(y_{i}-\hat{\hat{y}}_{i}\right)^{2}}{n-k-1}$. The denominator of this formula should read $n-k$ rather than $n-k-1$. 
The BASIC program LINFIT***, available in the C-E-I-R Multi-Access Computer Service, in order to obtain $\hat{\beta}$, inverts the matrix $A=\left[\begin{array}{cc}X^{\prime} X & X^{\prime} Y \\ Y^{\prime} X & \Sigma y_{i}^{2}\end{array}\right]$ whose inverse, if it exists, is

$$
\left[\begin{array}{cc}
\left(X^{\prime} X\right)^{-1}+\frac{\hat{\beta} \hat{\beta}^{\prime}}{\Sigma y_{i}^{2}-Y^{\prime} \hat{Y}} & \frac{-\hat{\beta}}{\Sigma y_{i}^{2}-Y^{\prime} \hat{Y}} \\
\frac{-\hat{\beta}^{\prime}}{\Sigma y_{i}^{2}-Y^{\prime} \hat{Y}} & \frac{1}{\Sigma y_{i}^{2}-Y^{\prime} \hat{Y}}
\end{array}\right]
$$

When $\hat{Y}=Y$, the matrix $A$ is singular. In the two test problems $\hat{Y}=Y$, and the matrix $A$, if it were formed in the computer without any rounding error, would be singular. But $A$, for $Y 1$ and $Y 2$, contains 14-digit numbers, whereas the GE 235 computer works with approximately nine-digit numbers, so that rounding of the elements of $A$ is inevitable, and the version of $A$ contained in the computer is not singular. An "inverse" was obtained, and from this $\hat{\beta}$ was immediately computed. Table 2 gives the results.

A third problem was used to test the program LINFIT***. Here we had

$$
X=\left[\begin{array}{lll}
1 & 0 & 5 \\
1 & 0 & 4 \\
1 & 2 & 1 \\
1 & 2 & 2
\end{array}\right], Y=\left[\begin{array}{l}
6 \\
5 \\
4 \\
5
\end{array}\right] \text {, and } A=\left[\begin{array}{rrrr}
4 & 4 & 12 & 20 \\
4 & 8 & 6 & 18 \\
12 & 6 & 46 & 64 \\
20 & 18 & 64 & 102
\end{array}\right]
$$

The last column (row) of $A$ is the sum of the three preceding columns (rows), so that $A$ is clearly singular. Unlike $Y 1$ and $Y 2$, this problem is such that the elements of $A$ have fewer than nine digits. An "inverse" was obtained by LINFIT***, however, and was printed as

$$
\left[\begin{array}{rrr}
a & a & a-a \\
a & a & a-a \\
a & a & a-a \\
-a & -a-a & a
\end{array}\right] \text { where } a=3.67091 \times 10^{7} .
$$

To obtain the inverse of $A$ in this program, the matrix command MAT $Q=\operatorname{INV}(A)$ is used. This command inverts the matrix $A$ by using an elimination method with row pivoting (Kurtz [28]). The method is described in section 1.2 of Stiefel [37].

LSCF - .*** is another BASIC least squares program available in the C-E-I-R Multi-Access Computer Service. Here the solution, $\hat{\beta}$, is obtained by inverting $X^{\prime} X$ and then post-multiplying the inverse by $X^{\prime} Y$. Table 2 shows that LSCF . - *** ranked below the other programs of this table. The inverse of $X^{\prime} X$ is obtained by using the matrix command INV, the same command as was used in LINFIT***.

The SIMEX-*** program originated at the Naval Ordnance Laboratory. The input required for this program was $X^{\prime} X$ and $X^{\prime} Y$, since SIMEX-*** solves $n$ equations in $n$ unknowns. An elimination algorithm is used to obtain the solution. The input for this program was limited to nine significant digits. Recalling the results of table 7 which gave the exact solution for the $Y 1$ problem when input data was rounded to nine digits, it is not surprising that the average number of correct digits for $Y 1$, reported in table 2 , is only 1.402 . This lies between the "accuracy" achieved by the nine-digit and ten-digit problems of table 7.

The BASIC program STAT21*** obtains $\left(X^{\prime} X\right)^{-1}$ and $\hat{\beta}$ by applying Jordan elimination to $X^{\prime} X$ and $X^{\prime} Y$; the results appear in table 2.

The LINFIT program included in table 1 is one of eighteen statistical routines described in On-line Analysis for Social Scientists by James R. Miller [32]. This library of routines exists in the Project MAC 7094 disk files. The two test problems were run on the LINFIT program on a timeshared computer via a remote console communicating with Project MAC. A description of Project 
MAC may be found in Crisman [11]. Miller states that "these routines may be used without extensive prior training in mathematics, statistics, or computer operations," but in view of LINFIT's poor performance on these two problems, it appears that there may be pitfalls in using this program. The method used by the LINFIT program is unknown. By conjecture, it has been included in this section among programs using elimination algorithms.

\section{Results from a Problem Having a Nonzero Standard Deviation}

In the two test problems, $Y 1$ and $Y 2$, treated thus far, the residual standard deviation was zero. A third test problem, $Y 1^{*}$, is one where the standard deviation is nonzero. This problem was run on five programs in both single and double precision to see whether the fact that a least squares fit has a standard deviation of zero might be a factor influencing the accuracy of computations.

The values of $Y 1^{*}$ were derived from the values of $Y 1$ by adding 2.0 to the $Y 1$ value when $x$ is even and subtracting 2.0 from the $Y 1$ value when $x$ is odd. The input for $Y 1 *$ is listed in table 9 . A fifth degree polynomial fit for the $Y 1^{*}$ problem has the solution (to 16 decimals)

$$
\hat{\beta}\left(Y 1^{*}\right)=\left[\begin{array}{c}
2.0459627329192547 \\
0.1815856777493606 \\
1.1701440301521066 \\
0.9870776685960425 \\
1.0003230582850989 \\
1.0000000000000000
\end{array}\right]
$$

with the residual standard deviation equal to 2.3251684 .

TABLE 8. Comparison of results from two problems: one with nonzero standard deviation (Y1*) and one with zero standard deviation (Y1)

All problems were run on the 1108 computer

Single Precision (8 Digits)

\begin{tabular}{|c|c|c|c|c|c|c|c|}
\hline \multirow[t]{2}{*}{ Program } & \multirow[t]{2}{*}{ Algorithm $^{\text {a }}$} & \multicolumn{2}{|c|}{$\begin{array}{l}\text { Average number of } \\
\text { correct digits }\end{array}$} & \multicolumn{2}{|c|}{ Rank } & \multicolumn{2}{|c|}{ Date of run } \\
\hline & & $Y 1^{*}$ & $Y 1$ & $Y 1 *$ & $Y 1$ & $Y 1^{*}$ & $Y 1$ \\
\hline BMDO2R... & $\mathrm{E}$ & 0.464 & -0.106 & 4 & 4 & $12-19-67$ & $12-13-67$ \\
\hline LSTSQ ....................... & HT & 3.485 & 4.528 & 2 & 1 & $10-15-68$ & $5-1-68$ \\
\hline MATH-PACK, ORTHLS.... & $\mathrm{OP}$ & 2.053 & 2.118 & 3 & 3 & $10-15-68$ & $4-12-68$ \\
\hline OMNITAB (Ortho) .............. & GS & 3.711 & 4.137 & 1 & 2 & $10-15-68$ & $10-18-67$ \\
\hline POLRG .......................... & $\mathrm{E}$ & -0.074 & -0.191 & 5 & 5 & $10-15-68$ & $10-7-68$ \\
\hline
\end{tabular}

Double Precision (18 Digits)

\begin{tabular}{|c|c|c|c|c|c|c|c|}
\hline \multirow[t]{2}{*}{ Program } & \multirow[t]{2}{*}{ Algorithma } & \multicolumn{2}{|c|}{$\begin{array}{l}\text { Average number of } \\
\text { correct digits }\end{array}$} & \multicolumn{2}{|c|}{ Rank } & \multicolumn{2}{|c|}{ Date of run } \\
\hline & & $Y 1^{*}$ & $Y 1$ & $Y 1 *$ & $Y 1$ & $Y 1^{*}$ & $Y 1$ \\
\hline BMDO2R..... & $\mathrm{E}$ & 9.657 & 9.645 & 4 & 4 & $4-17-68$ & $4-17-68$ \\
\hline LSTSQ $\ldots \ldots \ldots \ldots \ldots \ldots \ldots \ldots \ldots$ & HT & 13.913 & 14.643 & 1 & 1 & $10-16-68$ & $7-22-68$ \\
\hline MATH-PACK, ORTHLS .............................. & $\mathrm{OP}$ & 12.079 & 12.098 & 3 & 3 & $10-16-68$ & $10-16-68$ \\
\hline 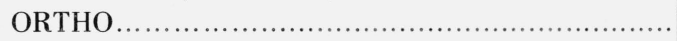 & GS & 13.136 & 13.188 & 2 & 2 & $3-27-68$ & $1-29-68$ \\
\hline POLRG ............ & $\mathrm{E}$ & 9.270 & 9.290 & 5 & 5 & $10-17-68$ & $10-7-68$ \\
\hline
\end{tabular}

${ }^{\mathrm{a}} \mathrm{E}=$ Elimination method; $\mathrm{GS}=\mathrm{Gram}-\mathrm{Schmidt}$ orthonormalization; HT=Orthogonal Householder transformations; OP=Orthogonal polynomials. 
TABLE 9. Input for fifth degree polynomials

\begin{tabular}{r|r|r|r}
\hline \hline$X$ & $Y 1$ & $Y 2$ & $Y 1^{*}$ \\
\hline & & & \\
1. & 1. & 1.00000 & 3. \\
2. & 6. & 1.11111 & 4. \\
3. & 63. & 1.24992 & 65. \\
4. & 364. & 1.42753 & 362. \\
5. & 1365. & 1.65984 & 1367. \\
6. & 3906. & 1.96875 & 3904. \\
7. & 9331. & 2.38336 & 9333. \\
8. & 19608. & 2.94117 & 19606. \\
9. & 37449. & 3.68928 & 37451. \\
10. & 66430. & 4.68559 & 66428. \\
11. & 111111. & 6.00000 & 111113. \\
12. & 177156. & 7.71561 & 177154. \\
13. & 271453. & 9.92992 & 271455. \\
14. & 402234. & 12.75603 & 402232. \\
15. & 579195. & 16.32384 & 579197. \\
16. & 813616. & 20.78125 & 813614. \\
17. & 1118481. & 26.29536 & 1118483. \\
18. & 1508598. & 33.05367 & 1508596. \\
19. & 2000719. & 41.26528 & 2000721. \\
20. & 2613660. & 51.16209 & 2613658. \\
& 3368421. & 63.00000 & 3368423. \\
\hline
\end{tabular}

Matrix $X^{\prime} X$

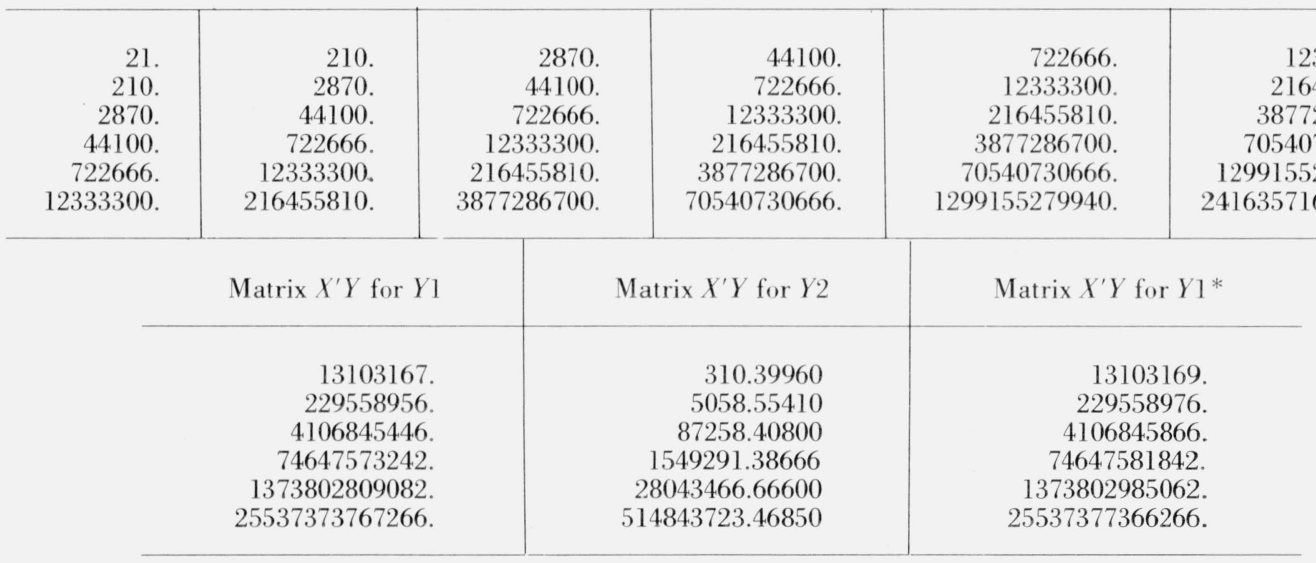

Table 8 summarizes the results, comparing the accuracy of the coefficients for $Y 1^{*}$ with the corresponding accuracy for $Y 1$. We see that the results for the two problems are quite similar, in both single and double precision. The largest differences occurred with the program LSTSQ in single precision, where the average number of correct digits was 4.528 for $Y 1$, and the average for $Y 1^{*}$ was 3.485 , a decrease of 1.043 .

On the basis of this comparison it appears that the fact that the standard deviation was zero in the test problems did not appreciably affect the accuracy of computations.

\section{Concluding Remarks}

(1) Computational procedures appropriate for desk calculators may be perilous for computers.

(2) Of the four procedures using floating-point arithmetic which were included in this study. orthogonal Householder transformations and Gram-Schmidt orthonormalization proved to be the best. Orthogonal polynomials ranked next. Elimination methods were the least successful but the most popular. The multiple precision integer arithmetic procedure using congruential methods was unique in obtaining exact solutions. 
(3) Some other algorithms apparently of high quality which have been published in the last few years were not included in this study. These include:
(a) Bauer [2],
(b) Björck and Golub [6],
(c) Björck [5].

Bauer [2] gives an ALGOL procedure using iterative refinement for finding the least squares solution of $X \beta=Y$, where $X$ is $n \times k(k \leqslant n)$ of rank $k$ and $Y$ is $n \times p$. The procedure is based on the decomposition of $X$ into UDR where $U$ is $n \times k$ with orthogonal columns, $D=\left(U^{\prime} U\right)^{-1}$, and $R$ is upper triangular. This decomposition yields a triangular system $R \beta=U^{\prime} Y$ which is solved by back substitution. The reduction to $R \beta=U^{\prime} Y$ is carried out by a Gaussian elimination scheme, but with a suitably weighted combination of rows used for elimination instead of a single row.

Björck and Golub [6] and Björck [5] (see also Björck [3], [4]) give two least squares algorithms with certain common features. Both take advantage of the fact that $X^{\prime} \delta=0$, where $\delta$ is the vector of residuals, to obtain the solution $\beta$ in $X \beta=Y$ from the augmented system of $n+k$ equations:

$$
\left[\begin{array}{cc}
I & X \\
X^{\prime} & 0
\end{array}\right]\left[\begin{array}{l}
\delta \\
\beta
\end{array}\right]=\left[\begin{array}{l}
Y \\
0
\end{array}\right]
$$

Both algorithms include $\delta$ as well as $\beta$ in the iterative refinement procedure.

The two algorithms are based on (i) orthogonal Householder transformations, and (ii) a modified Gram-Schmidt orthogonalization process.

Both the classical Gram-Schmidt orthogonalization process and the modified Gram-Schmidt orthogonalization process, as described by Björck [3], decompose the matrix $X$ into $Q R$ where $Q^{\prime} Q$ is diagonal and $R$ is upper triangular. In the classical procedure, at the $i$ th stage, the $i$ th column vector is made orthogonal to each of the $i-1$ previously orthogonalized column vectors; this is done for column indices $i=2,3, \ldots, k$. In the modified procedure which Björck uses, at the $i$ th stage, the $(k-i+1)$ column vectors indexed $i, i+1, \ldots, k$ are made orthogonal to the $(i-1)$-th column vector; this is done for column indices $i=2,3, \ldots ., k$. Jordan [27] shows why the modified procedure is superior to the classical procedure. Björck [3] states that his modified Gram-Schmidt procedure is equivalent to Bauer's method using weighted row combinations mentioned above. The algorithms for both the orthogonal Householder transformation method and the modified Gram-Schmidt method are generalized to handle the case where $X$ is of less than full rank. In this case linear constraints are entered.

The papers of Björck [3,5] and Björck and Golub [6] discuss the number of operations and the storage requirements of their least squares algorithms.

(4) Programmers who have been writing least squares programs, especially for statistical packages, have often not been taking advantage of the advances in this area made by numerical analysts in recent years.

(5) The importance of accumulating inner products in double precision cannot be overstressed. A number of recent papers on least squares computations have emphasized this point. These include Businger and Golub [8], Bauer [2], Golub and Wilkinson [22], Björck and Golub [6], and Björck [5]. On many third-generation computers which have double precision built into the hardware, double precision arithmetic is quite efficient.

(6) Iterative refinement is another valuable feature of recent algorithms. The three algorithms described in remark (3) above all include this feature. Four programs included in the present study (LSFITW***, LSTSQ, ORTHO, and ORTHOL) made effective use of iterative refinement. Golub and Wilkinson [22], who discuss this topic, also mention that the condition number of $X^{\prime} X$ is approximately the square of the condition number of $X$, so that it is advantageous to work with $X$ rather than $X^{\prime} X$ whenever possible. Moler [33] and Forsythe [19] discuss the details of iterative refinement in connection with solving $n \times n$ systems of linear equations. 
(7) The users of least squares programs can take certain precautionary steps to gain an awareness of whether or not a rounding error problem exists. Various suggestions were made in the previous studies of Cameron, Freund, Zellner and Thornber, and Longley. These suggestions included the following:

(a) Run test problems where the coefficients are known.

(b) Transform the data (e.g., by subtracting means).

(c) Do the calculations several times, scaled differently each time.

(d) Shuffle the columns of $X$ and run the problem more than once.

(e) Check whether $X^{\prime} \delta=0$.

(f) Use double precision arithmetic.

(8) Another check on the accuracy of least squares coefficients, suggested by Joseph M. Cameron, is the following. After carrying out the usual fit of $Y$ to $k$ independent variables, do a second fit, taking $\hat{Y}$ (the predicted values) and refitting to the $k$ original independent variables. If there were no rounding error at all, one would obtain exactly the same coefficients from the refit as from the original fit, and the standard deviation of the refit would be zero. The extent to which the second set of coefficients agrees with the original set can give one some information about the severity of rounding error.

A number of test problems were run on the 7094 and the 1108 in order to investigate the relationships among the coefficients of the original fit, those of the refit, and those one would obtain if there were no rounding error. The test problems consisted of 55 polynomials with various ranges of $x$, various degrees from 1 to 8 , and various coefficients. All 55 were run in both single and double precision on the 7094, and twelve of them were run in single and double precision on the 1108 .

In these test problems, the following result was obtained: If the coefficients from the refit (denoted by $\hat{b}$ ) agreed with the coefficients from the original fit $(\hat{\beta})$ to an average of more than three digits, and if the elements of $X^{\prime} X$ and $X^{\prime} Y$ can be represented in the computer without rounding, then the number of digits in $\hat{\beta}_{j}$ in agreement with $\hat{b}_{j}(j=1, \ldots ., k)$ was approximately the same as the number of correct digits in $\hat{\beta}_{j}$. More precisely, whenever the two conditions just stated were met, it was found (with one exception) that the number of digits in $\hat{\beta}_{j}$ in agreement with those of $\hat{b}_{j}(\mathrm{i})$ in double precision was within 1.0 of the number of correct digits in $\hat{\beta}_{j}$, and (ii) in single precision was within 2.0 of the number of correct digits in $\hat{\beta}_{j}$, for all $j$. The one exception occurred in a sixth degree polynomial with $x=-10$ (1) 10. In the double precision run the two sets of coefficients agreed to an average of about 12.5 digits, and the elements of $X^{\prime} X$ and $X^{\prime} Y$ had at most 13 significant digits; here $\hat{\beta}_{2}$ agreed with $\hat{b}_{2}$ to 16 digits but was correct only to about 13 digits.

(9) Efforts to provide comparative data on the amount of computer time required by the various programs included in this investigation, as well as comparisons of storage requirements, were unsuccessful. The programs which were included in this study originated from many sources, and they exhibited considerable variation with respect to what quantities were calculated as well as with respect to the methods of calculation. The program ALSQ, for example, at one end of the spectrum, calculated simply the coefficients, the residuals, the predicted values, and the residual sum of squares for the requested fifth degree polynomial. The single precision version of ALSQ required eight seconds to process both test problems on the 1108; the storage requirements were 709 memory cells for the code and 323 for the data. The double precision version of ALSQ processed both problems in seven seconds, requiring 715 memory cells for the code and 618 for the data. Nearer the other end of the spectrum was the Biomed program BMD05R. The output here consisted of the coefficients and their standard deviations for polynomial fits of degrees $1,2,3,4,5$, an analysis of variance for degrees $1,2,3,4,5$, predicted values and residuals for degree 5 , a plot of observed and predicted values for degree 5, and means and correlation coefficients of the input data. This program (computing some operations in double precision) required 20 seconds on the 1108 to process the two test problems; the storage requirements were 3,119 memory cells for the code and 15,168 for the data. It becomes evident that an intercomparison of running time among the different programs is not meaningful. 
Moreover, in repeated runs of a particular program there is fluctuation from run to run in the amount of time required. For example, on the same day three separate jobs were submitted to be run on OMNITAB (using the command POLYFIT) on the 1108, with the following|results:
(a) $Y 1$ alone:
8 seconds.
(b) $Y 2$ alone:
12 seconds.
(c) $Y 1$ and $Y 2$ together: 8 seconds.

The 1108 version of OMNITAB requires about 50,000 memory cells for storage. The run times given here include unknown components of time for operation of the computer system.

Although one would expect a double precision version of a particular program to require more time than a single precision version, there were several instances on the 1108 where double precision required less time than single precision.

It was outside the scope of this investigation to make a detailed comparison of algorithms with respect to efficiency of computation time and storage requirements. Similarly, no comparative examination of the outputs provided by the programs was made. Rather, this study focused attention on the performance of existing programs.

(10) In any mathematical calculation carried out on a computer, it is desirable to know whether an accurate solution has been obtained or whether the result of a calculation is contaminated by rounding error to such an extent that it is worthless. This goal has been achieved in some areas. Martin, Peters, and Wilkinson [31], in their paper giving an algorithm for solving $A x=b$, where $A$ is an $n \times n$ positive definite matrix and $b$ is an $n \times p$ matrix, state that their procedure "either produces the correctly rounded solutions of the equation $A x=b$ or indicates that $A$ is too ill-conditioned for this to be achieved without working to higher precision (or is possibly singular)." Similarly, Wilkinson's program [45] for the solution of an ill-conditioned system of equations $A x=b$, where $A$ is $n \times n$, "gives either a solution of the system which is correct to working accuracy or alternatively indicates that the system is too ill-conditioned to be solved without working to higher precision and may even be singular."

It appears that the goal set out above has now been achieved in the linear least squares program of Bjorck and Golub [6]. The authors state that their procedure may be used to compute accurate solutions and residuals to linear least squares problems, but that the procedure will fail when $X$ modified by rounding errors has less than full rank, and that it will also fail if $X$ is so illconditioned that there is no perceptible improvement in the iterative refinement. The user is easily informed of these situations.

I would like to express my appreciation to Joseph M. Cameron who suggested this investigation and made many valuable contributions, to Joan R. Rosenblatt for helpful discussions, and to Joseph Hilsenrath, Russell A. Kirsch, and Thomas Hoover for use of their time-shared computer facilities to run several problems. Thanks are due to Gene H. Golub, Stanford University, for his constructive remarks. The assistance of James Doyle, Univac Division, Sperry Rand Corporation, in debugging one program is also appreciated.

\section{Appendix A. Sources of the Programs, With Brief Descriptions}

ALSQ. A FORTRAN IV subroutine to solve the linear least squares problem, written by G. W. Stewart III, Union Carbide Corp., Oak Ridge, Tennessee (present address: The University of Texas, Austin, Texas). This program uses a modification of the Businger-Golub algorithm [8].

BMD02R, Stepwise Regression. One of the Biomedical Computer Programs, written in FORTRAN [15].

BMD03 R, Multiple Regression with Case Combinations. One of the Biomedical Computer Programs, written in FORTRAN [15].

BMD05R, Polynomial Regression. One of the Biomedical Computer Programs, written in FORTRAN [15]. 
DAM. A general purpose computer program for data processing and multiple regression, written in FORTRAN by Rudolf R. Rhomberg, Lorette Boissonneault, and Leonard Harris, International Monetary Fund [36].

DPVMTX. A double precision FORTRAN IV program for inverting a matrix or solving a set of linear equations. To a program from the SHARE library (7090-F1 3181INV2 Double Precision Matrix Inversion with Selective Pivot, written by A. R. Sadaka [26]), Sally T. Peavy, National Bureau of Standards, incorporated accuracy checks.

LINFIT. A program which fits a linear function to collected data via least squares. Optional constraints may be applied to the fitting coefficients to make them nonnegative, add to a constant, etc. One of eighteen statistical routines written by James R. Miller [32]. This library of routines exists in the Project MAC 7094 in the disk files of user number T169 2750.

LINFIT***. A program written in BASIC for linear least squares curve fitting and computing correlations. Origin: Dartmouth College, Hanover, N.H. Available in the C-E-I-R Multi-Access Computer Services library [10].

LSCF - ***. A least squares polynomial curve fitting subroutine written in BASIC. Origin: Dartmouth College, Hanover, N.H. Available in the C-E-I-R Multi-Access Computer Services library [10].

LSFITW***. A least squares curve fitting program written in BASIC. Adapted by John B. Shumaker, National Bureau of Standards, from Philip J. Walsh's ORTHO algorithm [42]. Available in the C-E-I-R Multi-Access Computer Services library [10].

LSTSQ. A FORTRAN IV subroutine which solves for $X$ the overdetermined system $A X=B$ of $m$ linear equations in $n$ unknowns for $p$ right-hand sides. Written by Peter Businger, Computation Center, University of Texas (present address: Bell Telephone Laboratories, Murray Hill, N.J.), using the Businger-Golub algorithm [8].

MATH-PACK, ORTHLS, Orthogonal Polynomial Least-Squares Curve Fitting. One of the Univac 1108 MATH-PACK programs, written in FORTRAN V [40].

MPR3, Stepwise Multiple Regression with Variable Transformations. A FORTRAN II program written by M. A. Efroymson, Esso Research and Engineering Co., Madison, N.J., using the Efroymson algorithm [16]. Available in the SHARE library: 7090-G2 3145MPR3 [26].

OMNITAB, a general-purpose computer program for statistical and numerical analysis. Developed at the National Bureau of Standards by Joseph Hilsenrath et al. [23]. Now available in an A. S. A. FORTRAN version, OMNITAB allows the user to communicate with a computer in an efficient manner by means of simple English sentences.

ORTHO. A program written by Philip J. Walsh, National Bureau of Standards (present address: University Computing Co., East Brunswick, N.J.), which uses a Gram-Schmidt orthonormalization process for least squares curve fitting. ORTHO exists as an ALGOL procedure [42], a FORTRAN program, a BASIC program (see LSFITW*** above), and as a routine of OMNITAB [23], where it is called by the commands FIT and POLYFIT.

ORTHOL. A modification of the Davis-Rabinowitz orthonormalization algorithm [12, 13, 14], written in FORTRAN II by James W. Longley, Bureau of Labor Statistics, Washington, D.C., and Roger A. Blau, Bureau of Labor Statistics and Carnegie-Mellon University, Pittsburgh, Pa. [30].

POLFIT. An anonymous program written in BASIC for least squares polynomial curve fitting using orthogonal polynomials.

POLRG, Polynomial Regression. One of the programs of the IBM System/360 Scientific Subroutine Package written in FORTRAN IV [24, 25].

SIMEX-***. A program written in BASIC for solving $n$ simultaneous equations in $n$ unknowns. Origin: Naval Ordnance Laboratory, Silver Spring, Md. Available in the C-E-I-R Multi-Access Computer Services library [10].

SOLVER. A FORTRAN program written by Morris Newman, National Bureau of Standards, for obtaining the exact solution of the system $A X=B$, or the inverse of a matrix $A$, by congruential methods [35]. The elements of $A$ and $B$ must be integers. 
SPVMTX. A single precision FORTRAN IV program for inverting a matrix or solving a set of linear equations. To a program from the SHARE library (7090-F1 3180INV1 Single Precision Matrix Inversion with Selective Pivoting, written by A. R. Sadaka [26]), Sally T. Peavy, National Bureau of Standards, incorporated accuracy checks.

STAT-PACK, GLH, General Linear Hypotheses. One of the Univac 1108 STAT-PACK programs, written in FORTRAN V [41].

STAT-PACK, REBSOM, Back Solution Multiple Regression. One of the Univac 1108 STATPACK programs, written in FORTRAN V [41].

STAT-PACK, RESTEM, Stepwise Multiple Regression. One of the Univac 1108 STAT-PACK programs, written in FORTRAN V [41].

STAT2 0***. A program written in BASIC for stepwise multiple linear regression. Written by Thomas E. Kurtz, Dartmouth College, Hanover, N.H. Available in the C-E-I-R Multi-Access Computer Services library [10].

STAT2 1***. A program written in BASIC for multiple linear regression with detailed output. Written by Gerald Childs, Dartmouth College, Hanover, N.H. Available in the C-E-I-R Multi-Access Computer Services library [10].

WRAP, Weighted Regression Analysis Program. A FORTRAN II program written by M. D. Fimple, Sandia Corp., Albuquerque, New Mexico. Available in the SHARE library: 7090-G2 3231 WRAP [26]. 
ALSQ

BETA-HAT (Y1)

1. 0228963

.99553592

.99941321

1.0001108

.99999613

1.0000000
AVERAGE $=4.098$

BMD02R

BETA-HAT (Y1)

$-17.13281$

39.34436

$-13.26675$

2. 92344

.89241

1.00212

$$
\begin{array}{r}
\text { COUNT } \\
-1.258 \\
-1.584 \\
-1.154 \\
-.284 \\
.968
\end{array}
$$$$
\text { 2. } 674
$$

AVERAGE $=-.106$

BMD03R

BETA-HAT (Y1)

394.23438

$-16.00000$

28. 00000

$-1.00000$

1. 00000

1. 00049

$$
\text { AVERAGE }=-.106
$$$$
\text { COUNT }
$$$$
-1.898
$$$$
-1.230
$$$$
-1.431
$$$$
-.301
$$$$
\text { 6. } 000
$$$$
\text { 3. } 310
$$

AVERAGE $=.742$

BMD03R

BETA-HAT (Y1)

5161.95310

40.000000

1.00

DAM
4.0000001 .50000000 89062500 99804688

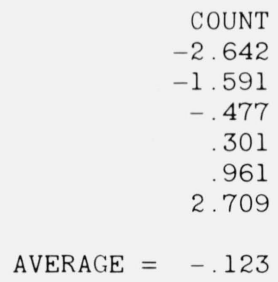

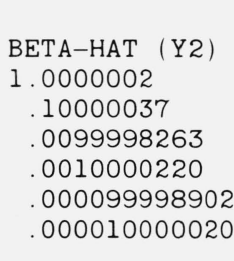
BETA-HAT (Y2)
.99954

10098775

0096306379

0010499504

000097199970

.000010055379

BETA-HAT (Y2)
1.04353
.10083
.01013
.00101
.00010
.00001

BETA-HAT (Y2)

1. 07353

10131836

010009766

00099945068

000097751617

0000099837780

AVERAGE $=$

7094

\section{EXAMPLE 3}

COUNT

1. 361

2. 081

1. 886

2. 000

2. 000

1. 000

AVERAGE $=1.721$

1108 EXAMPLE 4 COUNT

1. 134

1. 880

3. 010

3. 260

1. 648

2. 790

AVERAGE $=2.287$

BETA-HAT (Y2)

1. 000

101

00975

00103

0000982

0000100

7094

EXAMPLE 5

COUNT

4.000

2. 000

1. 602

1. 523

1. 745

3. 000

AVERAGE $=2.312$

1108

EXAMPLE 6 COUNT

4.003

2. 657

2. 074

1. 936

2. 182

2. 881 099779484 010084331 0098840467 0000099868524

AVERAGE $=-.264$

AVERAGE $=2.622$ 


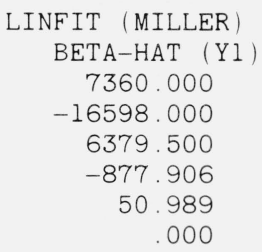

COUNT
-3.867
-4.220
-3.805
-2.944
-1.699
.000

AVERAGE $=-2.756$

LSTSQ

BETA-HAT (Yl)

99973875

1.0006891

99970413

1. 0000452

.99999718

1.0000001

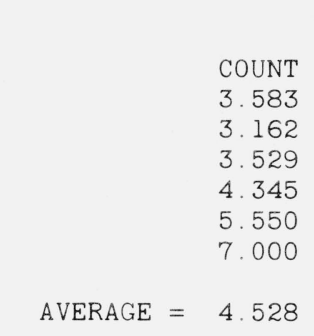

$$
\begin{aligned}
& \text { BETA-HAT (Y2) } \\
& 1.074 \\
& -.066 \\
& .074 \\
& -.008 \\
& .001 \\
& .000
\end{aligned}
$$

MATH-PACK 13.5, ORTHLS, ORTHOGONAL POLYNOMIAL CURVE FITTING BETA-HAT (Y1) .94458008

1. 1799316 91607666

1. 0135651 .99912310

1.0000196

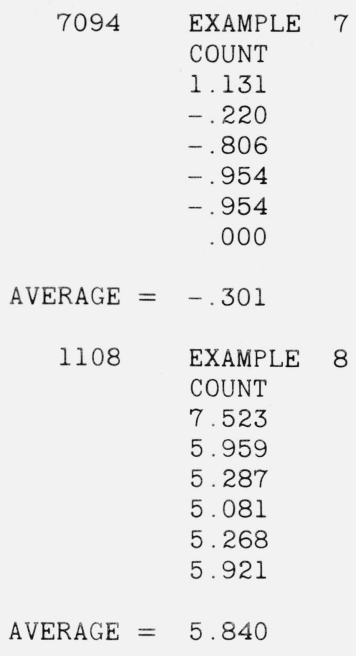

1108

EXAMPLE 9 COUNT

6.041

4.492 99999909

10000322

.0099984696

.0010002495

.000099983743

3. 815

3. 603

3.789

.000010000364
AVERAGE $=2.118$

AVERAGE $=4.363$

MPR3, STEPWISE MULTIPLE REGRESSION, SHARE LIBRARY 3145MPR3 BETA-HAT (Y1

$-20.24219$

COUNT

$$
-1.327
$$

43.49164

$-1.628$

$-14.37052$

$-1.187$

3. 03207 88800

$-.308$ .951

1.00219
BETA-HAT (Y2) .99933

1013436

009510736

.001065033

00009639953

.00001007054

AVERAGE $=-.140$

OMNITAB, USING THE MATRIX COMMANDS MTRANS, MMULT, INVERT

$$
\begin{gathered}
\text { BETA-HAT (YI) } \\
-91.999999 \\
136.00000 \\
-48.000000 \\
5.5000000 \\
.75000000 \\
1.0063477
\end{gathered}
$$

$$
\begin{aligned}
& \text { COUNT } \\
&-1.968 \\
&-2.130 \\
&-1.690 \\
&-.653 \\
& .602 \\
& 2.197 \\
& \text { AVERAGE }=-.607
\end{aligned}
$$

7094

\section{EXAMPLE 10}

COUNT

3.174

1. 872

1. 310

1. 187

1. 444

2. 152

AVERAGE $=1.856$

7094

EXAMPLE 11

COUNT

2. 658

1. 555

875

797

1. 125

1. 751

AVERAGE $=1.460$

1108

EXAMPLE 12 COUNT

2. 312

1. 145

647

628

939

1. 670

AVERAGE $=1.224$ 

BETA-HAT (YI) COUNT

$99780273 \quad 2.658$

$99932861 \quad 3.173$

1. 0001755

99998569

1. 0000004
OMNITAB, USING ORTHO SUBROUTINE

$\begin{array}{ll}1.0012817 & 2.892\end{array}$
3. 756

4.844

6.398

AVERAGE $=3.954$

$\begin{array}{lll} & 7094 & \text { EXAMPLE } 13 \\ \text { BETA-HAT (Y2) } & \text { COUNT } \\ .99999949 & 6.292 \\ .10000013 & 5.886 \\ .0099999756 & 5.613 \\ .0010000018 & 5.745 \\ .000099999866 & 5.873 \\ .000010000004 & 6.398\end{array}$

AVERAGE $=5.968$

2.189

3. 010

3.612

4.515

5.000

0000100

6. 495

AVERAGE $=4.137$

ORTHO, WITH RE-ORTHOGONALIZATION OMITTED BETA-HAT (Y1)

$-1216.5426$

2752.0557

$-1057.0931$

146.97336

$-7.3080225$

1. 1663037

COUNT

$-3.085$

$-3.439$

$-3.025$

$-2.164$

$-.919$

.779

AVERAGE $=-1.976$

ORTHOL

BETA-HAT (Y1)
.99784447
.98687472
1.0029743
.99961372
1.0000213
.99999960

COUNT

2.666

1. 882

2.527

3.413

4. 672

6. 398

AVERAGE $=3.593$
BETA-HAT (Y2) 99999990 099999700 010000125 00099998200 00010000109 0000099999778

$\begin{array}{cl}1108 \quad \text { EXAMPLE } 14 \\ \text { COUNT } \\ 7.000 \\ 5.523 \\ 4.903 \\ 4.745 \\ 4.963 \\ & 5.654 \\ & \\ \text { AVERAGE }= & 5.464\end{array}$

BETA-HAT (Y2) 98419483 13523918

$-.0034660707$ .0028495983

$-.0000049256487$ 000012094996

1108

EXAMPLE 15 COUNT

1.801 .453

$-.129$

$-.267$

$-.021$

.679

AVERAGE $=.419$

1108 EXAMPLE 16 COUNT

8.000

5. 654

5. 387

5.461

5. 886

6.796

.0000000

.00099999654

00010000013

0000099999984
010000041

AVERAGE $=6.197$

1108 EXAMPLE 17 COUNT

1. 807

3.046

1. 834

2. 266

2. 066

2. 659

57484

1. 165

3.373

000099141363

000010021910

AVERAGE $=-.191$

SPVMTX

BETA-HAT (Y1)

64. 191025

$-134.65426$

51.859977

$-5.8942945$

1. 3872223

.99232943

BETA-HAT (Y2)
1.0014403
.097101737
.011047948
.00086162069
.00010761766
.0000098514820

AVERAGE $=2.280$

1108

EXAMPLE 18

COUNT

2. 842

AVERAGE $=-.658$

1. 538

.980

.859

1. 118

1. 828

2. 115

$-2.132$

1.706

0000098514820

AVERAGE $=1.527$ 
STAT-PACK 8.13, GLH, GENERAL LINEAR HYPOTHESIS BETA-HAT (YI)

$-11.970093$

27. 245361

COUNT

$-1.113$

$-8.5661011$

$-1.419$

$-.981$

2. 2717514

$-.104$

92961073

1. 152

1. 0013784

2.861

BETA-HAT (Y2)

99989064

10018034

0099404901

.0010073970

000099610348

000010007344

AVERAGE $=.066$

STAT-PACK 9.2, REBSOM, BACK SOLUTION MULTIPLE REGRESSION

BETA-HAT (Y1)

$-11.97009$

27.24536

$-8.56610$

1.113

BETA-HAT (Y2)

2. 27175

.92961

1.00138

$\begin{array}{rc}\text { COUNT } & \text { BETA-HAT (Y2) } \\ -1.113 & .9998906 \\ -1.419 & .1001803 \\ -.981 & .009940490 \\ -.104 & .001007397 \\ 1.152 & .00009961035 \\ 2.860 & .00001000734\end{array}$

AVERAGE $=$

.066

$\begin{aligned} 1108 & \text { EXAMPLE } 19 \\ & \text { COUNT } \\ & 3.961 \\ & 2.744 \\ & 2.225 \\ & 2.131 \\ & 2.409 \\ & 3.134 \\ & \\ \text { AVERAGE }= & 2.767\end{aligned}$

1108

EXAMPLE 20 COUNT

3. 961

2.744

2. 225

2. 131

2. 409

3.134

AVERAGE $=2.767$

STAT-PACK 9.1, RESTEM, STEPWISE MULTIPLE REGRESSION

BETA-HAT (YI)

$-1.5703125$

7.5583214

COUNT

BETA-HAT (Y2)

$-.410$

1. 0002102

$-.817$

$-1.5695286$

$-.410$

.099611598

1. 3551083

.450

010136487

.97985181

1.696

00098222795

1.0004015

3. 396

00010097076

.0000099811599

AVERAGE =

.651

AVERAGE $=2.407$

WRAP, WEIGHTED REGRESSION, SHARE LIBRARY 323IWRAP

BETA-HAT (Y1)
2991622 .
-6065892.
2218821.
-296194.5
16462.20
-322.5731

COUNT

$-6.476$

$-6.783$

$-6.346$

$-5.472$

$-4.216$

$-2.510$

AVERAGE $=-5.300$

DETAILS FOR TABLE 2 -- SINGLE PRECISION (9 DIGITS)
LINFIT

BETA-HAT (Y1)

2. 76639

$-2.72473$

2. 38633

.812925

1.01048

999793

LSCF

BETA-HAT (YI)

$-5.5$

$-12$

$-3$

.957031

.999023
AVERAGE $=.905$

COUNT

$-.247$

$-.571$

$-.142$

.728

1.980

3. 684

AVERAGE =

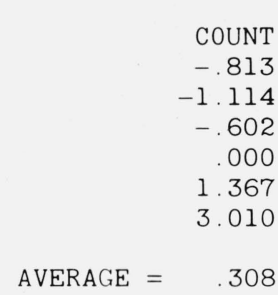

BETA-HAT (Y2)

$-33.84546$

71.54880

$-26.16913$

3. 493256

$-.1936966$

.003812985
1108 EXAMPLE 21 COUNT

3.677

2. 411

1.865

1. 750

2.013

2.725

7094

EXAMPLE 22

COUNT

$-1.542$

$-2.854$

$-3.418$

$-3.543$

$-3.287$

$-2.580$

AVERAGE $=-2.871$

EXAMPLE I

COUNT

4. 222

2. 908

2. 347

2. 223

2. 479

3.187

AVERAGE $=2.894$

BETA-HAT (Y2)

999893

099762

00991058

000970840

0000993013

.00000997260

EXAMPLE 2

COUNT

3. 971

2. 623

2. 049

1. 535

2. 156

2. 562

AVERAGE $=2.483$ 
DETAILS FOR TABLE 2 -- SINGLE PRECISION (9 DIGITS)

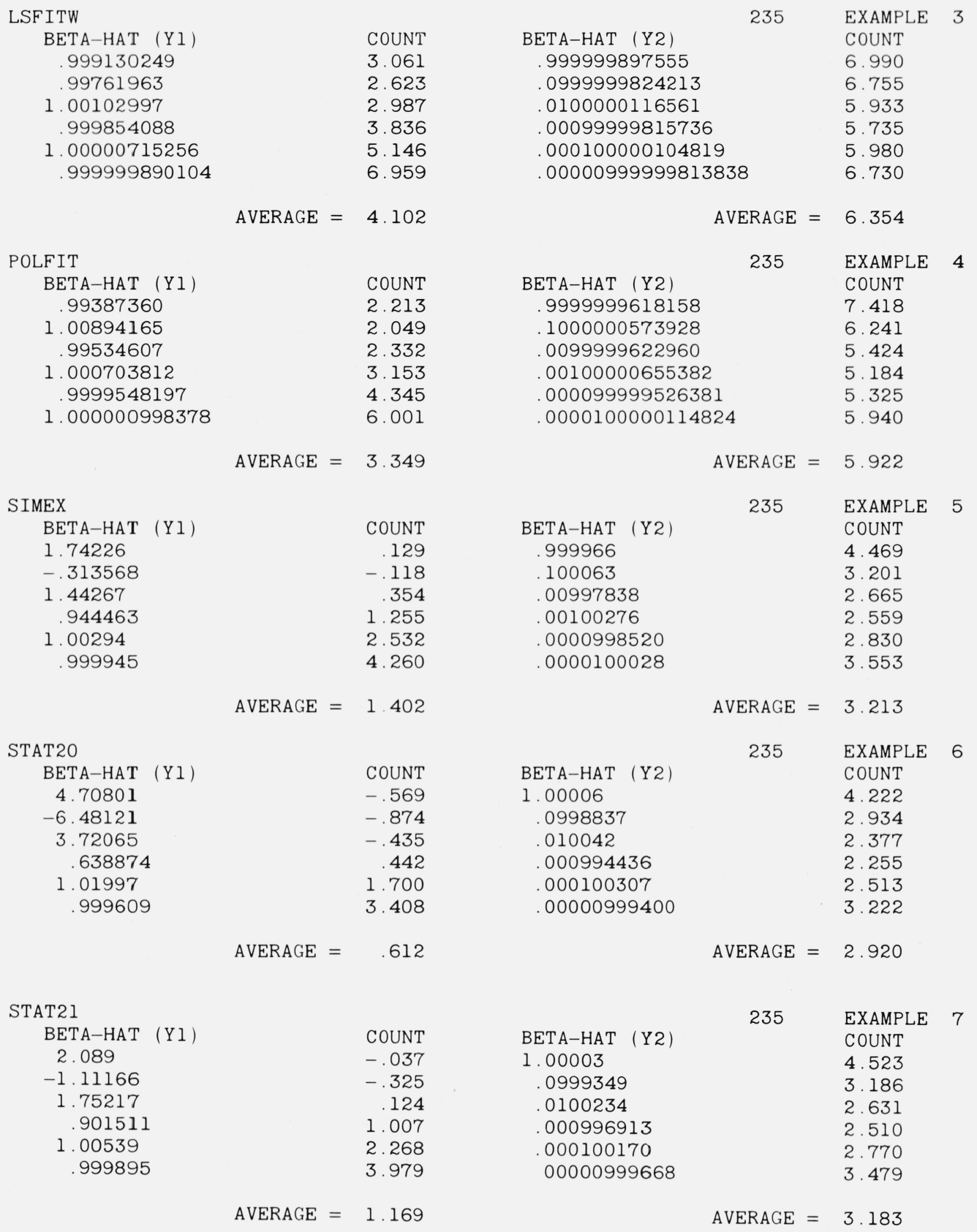




\section{BMD05R}

BETA-HAT (YI) COUNT

1.0000003 99999920

1.0000002 99999996 9999999 99999999

\subsection{3}

6.097

6. 699

7.398

7.000

8. 000

AVERAGE $=6.953$

DPVMTX
BETA-HAT (YI)
1. 000000206882520 .9999995965200948
1. 000000145134836 9999999808270597
1. 000000001057576 .9999999999793303
AVERAGE $=7.882$

$\begin{array}{ccl} & 7094 & \text { EXAMPLE } \\ \text { BETA-HAT (Y2) } & & \text { COUNT } \\ .99999998 & 7.699 \\ .10000004 & 6.398 \\ .0099999798 & & 5.695 \\ .0010000031 & 5.509 \\ .000099999792 & 5.682 \\ .000010000004 & & 6.398 \\ & \text { AVERAGE }=6.230\end{array}$

\begin{tabular}{|c|c|}
\hline 1107 & EXAMPLE \\
\hline BETA-HAT (Y2) & COUNT \\
\hline 1.000000000006017 & 11.221 \\
\hline .09999999998899705 & 9.958 \\
\hline 01000000000383598 & 9.416 \\
\hline 0009999999995039349 & 9.304 \\
\hline .0001000000000269390 & 9.570 \\
\hline .000009999999999479781 & 10.284 \\
\hline
\end{tabular}

AVERAGE $=9.959$

DETAILS FOR TABLE 4 -- DOUBLE PRECISION (18 DIGITS)

ALSQ

BETA-HAT (Y1)

1. 000000000005630 9999999999933983

1. 000000000002151 9999999999997248

1.000000000000015 9999999999999997

AVERAGE $=12.667$

BMD02R

BETA-HAT (YI)

COUNT .9999999968749762

1.000000006749235 9999999974683392

1. 000000000342994 9999999999807494

1. 000000000000381
COUNT

11. 249

11. 180

11.667

12.560

13.824

15.520
8. 171

8.597

9. 465

10.716

12.419

$\begin{array}{lll}\text { BETA-HAT (Y2) } 1108 & \begin{array}{l}\text { EXAMPLE } \\ \text { COUNT }\end{array} \\ 1.00000000000000000 & 18.000 \\ .0999999999999999140 & 15.065 \\ .0100000000000000339 & 14.470 \\ .000999999999999995720 & 14.368 \\ .000100000000000000224 & 14.650 \\ .00000999999999999999580 & 15.376\end{array}$

AVERAGE $=15.322$

BETA-HAT (Y2)

1108

EXAMPLE 2

1.000000000000007

COUNT

09999999999998616

01000000000000481

14.155

AVERAGE $=9.645$

BMD05R

BETA-HAT (Y1)

COUNT

1. 00000000634827302 .999999987016701379

1. 00000000476533960 .999999999362724245

1. 00000000003545420 999999999999302617

AVERAGE $=9.368$

DPVMTX

BETA-HAT (Y1) COUNT .9999999971390853

1. 000000005557233 9999999980049357

1. 000000000263132 9999999999855033

1. 000000000000283 $0009999999999993756 \quad 12.205$ $0001000000000000339 \quad 12.470$ $000009999999999999342 \quad 13.182$

$$
\text { AVERAGE }=12.865
$$

\begin{tabular}{|c|c|}
\hline BETA-HAT & COU \\
\hline 1.00000000000007691 & 13.11 \\
\hline 0999999999998442950 & 11.80 \\
\hline 0100000000000568478 & 11.245 \\
\hline 000999999999992425020 & 11.12 \\
\hline 000100000000000420311 & 1 . \\
\hline 00000999999999999174930 & \\
\hline
\end{tabular}

$$
\text { AVERAGE }=11.791
$$

$\begin{array}{ccc}\text { BETA-HAT (Y2) } & 1108 & \begin{array}{l}\text { EXAMPLE } \\ \text { COUNT }\end{array} \\ .9999999999999998 & & 15.693 \\ .1000000000000033 & & 13.481 \\ .0099999999999983^{r} & & 12.790 \\ .0010000000000002 & & 12.607 \\ .0000999999999999 \varepsilon & 7 & 12.826 \\ .00001000000000000 & 1 & 13.509\end{array}$

AVERAGE $=13.484$ 
DETAILS FOR TABLE 4 -- DOUBLE PRECISION (18 DIGITS)

LSTSQ

BETA-HAT (Y1) 999999999999999464 999999999999943084

1. 00000000000004282 999999999999991067

1. 00000000000000068 999999999999999985

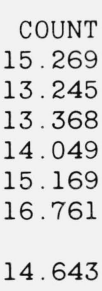

COUNT

13.368

14.049

\section{AVERAGE $=14.643$}

MATH-PACK 13.5 , ORTHLS, ORTHOGONAL POI

BETA-HAT (YI) 999999999992709829

1. 00000000001946887 999999999991466379

1. 00000000000133404 999999999999915178

1. 00000000000000188

$$
\begin{array}{r}
\text { COUNT } \\
11.137 \\
10.711 \\
11.069 \\
11.875 \\
13.071 \\
14.727
\end{array}
$$

AVERAGE $=12.098$

ORTHO

BETA-HAT (Y1) 999999999997051246

1. 00000000000051159

1. 00000000000034817 999999999999897859

1. 00000000000000760 999999999999999820

$$
\text { AVERAGE }=13.188
$$

ORTHO, WITH RE-ORTHOGONALIZATION OMITTED

BETA-HAT (Y1)

COUNT 999999858679196051

1. 00000031785242527 999999878054865120

1. 00000001679285067 999999999045590670

1. 00000000001908297

6.850

6. 498

6.914

7.775

9.020

10.719

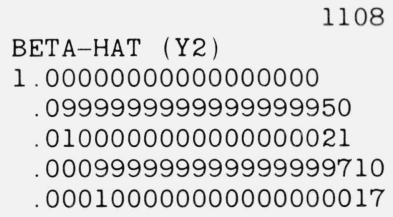

COUNT

18.000

16.284

15.683

15.538

15.771

16.480

$$
\text { AVERAGE }=16.293
$$

POLYNOMIAL

L CURVE FITTING 1108

BETA-HAT (Y2)

.999999999999999901

\begin{tabular}{|c|c|}
\hline 1108 & EXAMPLE \\
\hline 3ETA-HAT (Y2) & COUNT \\
\hline 999999999999999946 & 16.242 \\
\hline 0999999999999999970 & 16.488 \\
\hline 0100000000000000078 & 15.109 \\
\hline 000999999999999998330 & 14.777 \\
\hline .000100000000000000121 & 14.918 \\
\hline 00000999999999999999720 & 15.550 \\
\hline
\end{tabular}

EXAMPLE 6 100000000000000225 COUNT

14.648
0 $00999999999999988980 \quad 13.958$ $00100000000000001872 \quad 13.728$ $\begin{array}{lll}.0000999999999999987360 & 13.898\end{array}$ $0000100000000000000292 \quad 14.535$

$$
\text { AVERAGE }=14.461
$$

AVERAGE $=15.514$

\begin{tabular}{lrr} 
BETA-HAT $\quad$ (Y2) & 1108 & \multicolumn{1}{c}{ EXAMPLE } \\
COUNT & 8 \\
.999999999998151750 & 11.733 \\
.00000000004101749 & 10.387 \\
.00999999999843660520 & 9.806 \\
.00100000000021433129 & 9.669 \\
.0000999999999878591650 & 9.916 \\
.0000100000000002421209 & 10.616
\end{tabular}

$$
\text { AVERAGE }=7.963
$$

$$
\text { AVERAGE }=10.354
$$

ORTHOL

BETA-HAT (Y1) 999999999999754768

1. 00000000000255572 999999999999179468

1. 00000000000011276 999999999999993318

1. 00000000000000014

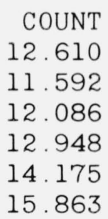

AVERAGE $=13.212$

$\begin{array}{ll}\text { BETA-HAT (Y2) } 1108 & \begin{array}{l}\text { EXAMPLE } \\ \text { COUNT }\end{array} \\ 1.00000000000000000 & 18.000 \\ .100000000000000036 & 15.444 \\ .00999999999999998660 & 14.873 \\ .00100000000000000191 & 14.719 \\ .0000999999999999998880 & 14.950 \\ .0000100000000000000023 & 15.640\end{array}$

AVERAGE $=15.604$

$\begin{array}{cccc}\text { POLRG, IBM SYSTEM/360 SCIENTIFIC SUBROUTINE PACKAGE } & 1108 & \text { EXAMPLE } 10 \\ \text { BETA-HAT (Y1) } & \text { COUNT } & \text { BETA-HAT (Y2) } & \text { COUNT } \\ 1.00000011110114428 & 6.954 & 1.00000000000136645 & 11.864 \\ .999999990457514712 & 8.020 & .0999999999999411990 & 12.231 \\ 1.00000000321449412 & 8.493 & .0100000000000305940 & 11.514 \\ .999999999544922239 & 9.342 & .000999999999995547370 & 11.351 \\ 1.00000000002322160 & 10.634 & .000100000000000227181 & 11.644 \\ .999999999999491835 & 12.294 & .00000999999999999416480 & 12.234\end{array}$




$\begin{array}{rccc}\text { STAT-PACK 9.1, RESTEM, STEPWISE MULTIPLE REGRESSION } & 1108 & \text { EXAMPLE } 11 \\ \text { BETA-HAT (Y } 1) & \text { COUNT } & \text { BETA-HAT (Y2) } & \text { COUNT } \\ 1.00000000453928806 & 8.343 & 1.00000000000004872 & 13.312 \\ .999999990379828084 & 8.017 & .0999999999999056810 & 12.025 \\ 1.00000000358299878 & 8.446 & .0100000000000336338 & 11.473 \\ .999999999516629162 & 9.316 & .000999999999995591090 & 11.356 \\ 1.00000000002705048 & 10.568 & .000100000000000241649 & 11.617 \\ .999999999999465664 & 12.272 & .00000999999999999530180 & 12.328 \\ & & & \text { AVERAGE }=12.019\end{array}$

DETAILS FOR TABLE 5 -- SINGLE PRECISION (8 DIGITS), WITH INNER PRODUCTS ACCUMULATED IN DOUBLE PRECISION ( 18 DIGITS)

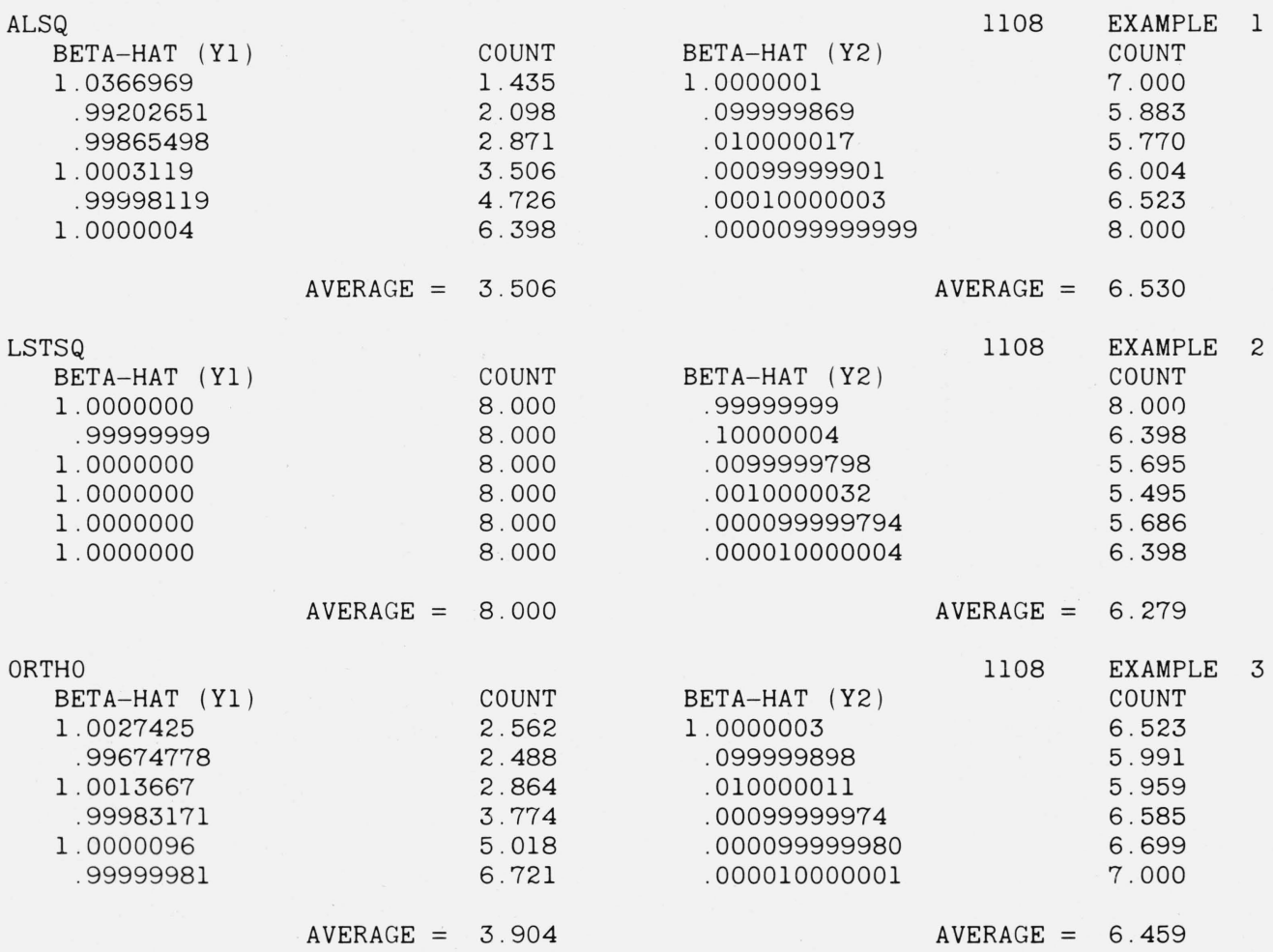

DETAILS FOR TABLE 6 -- MULTIPLE PRECISION INTEGER ARITHMETIC

$\begin{array}{lr}\text { SOLVER } & \text { COUNT } \\ \text { BETA-HAT (Y1) } & 18.000 \\ 1.00000000000000000 & 18.000 \\ 1.00000000000000000 & 18.000 \\ 1.00000000000000000 & 18.000 \\ 1.00000000000000000 & 18.000 \\ 1.00000000000000000 & 18.000 \\ 1.00000000000000000 & \\ \text { AVERAGE }= & 18.000\end{array}$

\begin{tabular}{|c|c|}
\hline 1108 & EXAMPLE \\
\hline BETA-HAT (Y2) & COUNT \\
\hline .999999999999999995 & 17.159 \\
\hline 0999999999999999995 & 17.187 \\
\hline .00999999999999999996 & 17.169 \\
\hline .000999999999999999996 & 17.294 \\
\hline .0000999999999999999996 & 17.276 \\
\hline .0000100000000000000000 & 18.000 \\
\hline
\end{tabular}




\section{References}

[1] Anscombe, F. J., Topics in the investigation of linear relations fitted by the method of least squares, Journal of the Royal Statistical Society, Series B, 29, p. 1-52, 1967.

[2] Bauer, F. L., Elimination with weighted row combinations for solving linear equations and least squares problems, Numerische Mathematik 7, p. 338-352, 1965.

[3] Björck, Ake, Solving linear least squares problems by Gram-Schmidt orthogonalization, BIT (Nordisk tidskrift for informations-behandling), 7, p. 1-21, 1967.

[4] Björck, Ake, Iterative refinement of linear least squares solutions, I, BIT 7, p. 257-278, 1967.

[5] Björck, Ake, Iterative refinement of linear least squares solutions, II, BIT 8, p. 8-30, 1968.

[6] Björck, Ake, and Golub, Gene, ALGOL Programming, Contribution No. 22: Iterative refinement of linear least square solutions by Householder transformation, BIT 7, p. 322-337, 1967.

[7] Bright, J. W., and Dawkins, G. S., Some aspects of curve fitting using orthogonal polynomials, Industrial and Engineering Chemistry Fundamentals 4, p. 93-97, 1965.

[8] Businger, Peter, and Golub, Gene H., Linear least squares solutions by Householder transformations, Numerische Mathematik 7, p. 269-276, 1965.

[9] Cameron, J. M., Some examples of the use of high speed computers in statistics, Proceedings of the First Conference on the Design of Experiments in Army Research, Development and Testing, Office of Ordnance Research, Report No. 57-1, p. 129-135, 1957.

[10] C-E-I-R Multi-Access Computer Services, Library programs documentation, MAC 71-7-1, Nov. 17, 1967. Addendum, MAC 71-7-1, A 12-368, Mar. 1, 1968.

[11] Crisman, P. A., (ed.), The compatible time-sharing system: A programmer's guide, second edition, M. I. T. Computation Center, M. I. T. Press, Massachusetts Institute of Technology, Cambridge, Mass., 1965.

[12] Davis, Philip J., Orthonormalizing codes in numerical analysis, ch. 10 in [39], 1962.

[13] Davis, Philip, and Rabinowitz, Philip, A multiple purpose orthonormalizing code and its uses, Journal of the Association for Computing Machinery 1, p. 183-191, 1954.

[14] Davis, Philip J., and Rabinowitz, Philip, Advances in orthonormalizing computation, in Franz L. Alt (ed.), Advances in Computers, p. 55-133, Vol. 2, (Academic Press, New York, 1961).

[15] Dixon, W. J., (ed.), BMD Biomedical Computer Programs, Health Sciences Computing Facility (University of California, Los Angeles, 1964. Revised 1965 and 1967).

[16] Efroymson, M. A., Multiple regression analysis, in Anthony Ralston and Herbert S. Wilf (editors), Mathematical Methods for Digital Computers, Ch. 17, Vol. 1, pp. 191-203 (John Wiley \& Sons, New York, N.Y. 1960).

[17] Fettis, Henry E., and Caslin, James C., Eigenvalues and eigenvectors of Hilbert matrices of order 3 through 10, Mathematics of Computation 21, p. 431-441, 1967.

[18] Forsythe, George E., Generation and use of orthogonal polynomials for data-fitting with a digital computer, Journal of the Society for Industrial and Applied Mathematics 5, p. 74-88, 1957.

[19] Forsythe, George E., Today's computational methods of linear algebra, SIAM Review 9, p. 489-515, 1967.

[20] Freund, R. J., A warning of roundoff errors in regression, The American Statistician 17, p. 13-15, 1963.

[21] Golub, G., Numerical methods for solving linear least squares problems, Numerische Mathematik 7, p. 206-216, 1965.

[22] Golub, G. H., and Wilkinson, J. H., Note on the iterative refinement of least squares solution, Numerische Mathematik 9 , p. 139-148, 1966.

[23] Hilsenrath, Joseph, Ziegler, Guy G., Messina, Carla G., Walsh, Philip J., and Herbold, Robert, OMNITAB, A Computer Program for Statistical and Numerical Analysis, National Bureau of Standards Handbook 101, U. S. Government Printing Office, Washington, D.C., 1966. Reissued Jan. 1968, with corrections.

[24] IBM Application Program, System/360 Scientific Subroutine Package (360A-CM-03X) Version III, Application Description, H20-0166-5, International Business Machines Corp., 1968.

[25] IBM Application Program, System/360 Scientific Subroutine Package (360A-CM-03X) Version III, Programmer's Manual, H20-0205-3, International Business Machines Corp., 1968.

[26] IBM Systems Reference Library, Catalog of programs for IBM 704-709-7040-7044-7090 and 7094 Data Processing Systems, Form C20-1604-3, International Business Machines Corp., December 1965.

[27] Jordan, T. L., Experiments on error growth associated with some linear least-squares procedures, Mathematics of Computation 22, p. 579-588, 1968.

[28] Kurtz, Thomas E., personal communication, 1968.

[29] Longley, James W., An appraisal of least squares programs for the electronic computer from the point of view of the user, Journal of the American Statistical Association 62, p. 819-841, 1967.

[30] Longley, James W., personal communication, 1968.

[31] Martin, R. S., Peters, G., and Wilkinson, J. H., Iterative refinement of the solution of a positive definite system of equations, Numerische Mathematik 8, p. 203-216, 1966.

[32] Miller, James R., On-line analysis for social scientists, MAC-TR-40, Project MAC, Massachusetts Institute of Technology, Cambridge, Mass., 1967. 
[33] Moler, Cleve B., Iterative refinement in floating point, Journal of the Association for Computing Machinery 14, p. 316-321, 1967.

[34] Newman, Morris, Matrix computations, ch. 6 in [39], 1962.

[35] Newman, Morris, Solving equations exactly, J. Res. NBS 71B (Math. and Math. Phys.), No. 4, $171-179.1967$.

[36] Rhomberg, Rudolf R., Boissonneault, Lorette, and Harris, Leonard, A general purpose computer program for data processing and multiple regression (DAM), Revision 3, Research and Statistics Department, International Monetary Fund, Washington, D.C., Sept. 1965.

[37] Stiefel,-Eduard L., An Introduction to Numerical Mathematics, (Academic Press, New York, 1963).

[38] Taussky, Olga, Some topics concerning bounds for eigenvalues of finite matrices, ch. 8 in [39], 1962.

[39] Todd, John (ed.), Survey of Numerical Analysis (McGraw-Hill Book Co., New York, 1962).

[40] Univac 1108 Multi-Processor System, MATH-PACK Programmers Reference, UP-7542, Univac Division of Sperry Rand Corporation, 1967.

[4.1] Univac 1108 Multi-Processor System, STAT-PACK Programmers Reference, UP-7502, Univac Division of Sperry Rand Corporation, 1967.

[42] Walsh, Philip J., Algorithm 127, ORTHO, Communications of the ACM 5, p. 511-513, 1962.

[43] Wilkinson, J. H., Rounding Errors in Algebraic Processes (Prentice-Hall, Englewood Cliffs, N.J., 1963).

[44] Wilkinson, J. H., The Algebraic Eigenvalue Problem (Clarendon Press, Oxford, 1965).

[45] Wilkinson, J. H., The solution of ill-conditioned linear equations, in Anthony Ralston and Herbert S. Wilf (editors), Mathematical Methods for Digital Computers, ch. 3, p. 65-93, Vol. 2, (John Wiley \& Sons, New York, 1967).

[46] Zellner, A., and Thornber, H., Computational accuracy and estimation of simultaneous equation econometric models, Econometrica 34, pp. 727-729, 1966.

(Paper 73B2-289) 